\title{
tRNA-modifying enzyme mutations induce codon-specific mistranslation and protein aggregation in yeast
}

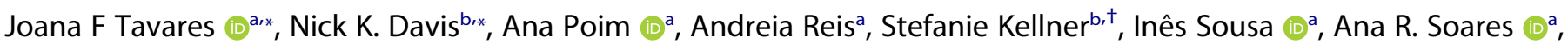 \\ Gabriela M R Moura ${ }^{a}$, Peter C Dedon (10 ${ }^{\mathrm{b}, \mathrm{c}}$, and Manuel Santos ${ }^{\mathrm{a}}$
}

aDepartment of Medical Sciences, Institute of Biomedicine - iBiMED, University of Aveiro, Aveiro, Portugal; ${ }^{b}$ Department of Biological Engineering, Massachusetts Institute of Technology - MIT, Cambridge, US; 'Singapore-MIT Alliance for Research and Technology, Campus for Research Excellence and Technical Enterprise - CREATE, Singapore

\begin{abstract}
Protein synthesis rate and accuracy are tightly controlled by the cell and are essential for proteome homoeostasis (proteostasis); however, the full picture of how mRNA translational factors maintain protein synthesis accuracy and co-translational protein folding are far from being fully understood. To address this question, we evaluated the role of 70 yeast tRNA-modifying enzyme genes on protein aggregation and used mass spectrometry to identify the aggregated proteins. We show that modification of uridine at anticodon position 34 (U34) by the tRNA-modifying enzymes Elp1, Elp3, Sml3 and Trm9 is critical for proteostasis, the mitochondrial tRNA-modifying enzyme SIm3 plays a fundamental role in general proteostasis and that stress response proteins whose genes are enriched in codons decoded by tRNAs lacking $\mathrm{mcm}^{5} \mathrm{U}_{34}, \mathrm{mcm}^{5} \mathrm{~s}^{2} \mathrm{U}_{34}, \mathrm{ncm}^{5} \mathrm{U}_{34}, \mathrm{ncm}^{5} \mathrm{Um}_{34}$, modifications are overrepresented in protein aggregates of the ELP1, SLM3 and TRM9 KO strains. Increased rates of amino acid misincorporation were also detected in these strains at protein sites that specifically mapped to the codons sites that are decoded by the hypomodified tRNAs, demonstrating that $U_{34}$ tRNA modifications safeguard the proteome from translational errors, protein misfolding and proteotoxic stress.
\end{abstract}

\section{ARTICLE HISTORY}

Received 14 July 2020

Revised 31 August 2020

Accepted 1 September 2020

\section{KEYWORDS}

(5-10) tRNA modifying enzymes; protein aggregation; yeast; tRNA proteome

ARTICLE HISTORY Received 14 July 2020; Revised 31 August 2020; Accepted 1 September 2020

\section{Introduction}

Transfer RNAs (tRNAs) are adapter molecules responsible for decoding mRNA into protein, playing critical roles in gene expression. In yeast, 42 different cytoplasmic tRNAs ( 1 initiator and 41 elongator tRNAs), encoded by 275 tRNA genes, read the 61 sense codons that code for the 20 universal amino acids [1-4]. The degeneracy of this code - more than one codon correspond to one single amino acid - is possible due to the flexibility of anticodon-codon pairing rules at the third codon position, which allows one tRNA molecule to decode more than one codon. This flexibility allows the first base of the anticodon (position 34 or the 'wobble' position) to interact with more than one base at the third position of the codon [5], according to wobble-base pairing rules. This complexity is modulated by enzymatic modification of tRNA anticodon nucleosides, particularly in positions 34 and 37 , that differentiate translationally the codons encoding the same amino acid $[5,6]$.

The first evidence that anticodon modifications modulate the recognition of specific codons was obtained for inosine (I) at position 34 and $\mathrm{N}^{6}$-isopentenyladenosine $\left(\mathrm{i}^{6} \mathrm{~A}\right)$ at position
37 (A3' adjacent to the anticodon) $[7,8]$. Unmodified $\mathrm{U}_{34}$ was initially believed to recognize both $A$ and $G$ at the third position of the codon [5]. Subsequent studies revealed it could recognize all four bases at the third codon position, especially in mitochondrial translational systems $[9,10]$, while its modification restricted recognition to only $\mathrm{A}$ and/or G. These findings began to functionally explain the abundance of $U_{34}$ modifications in cytoplasmic tRNAs [11]: in two-codon sets, modifications function to enhance discrimination between cognate pyrimidine-ending and noncognate purine-ending codons. This codon decoding regulatory paradigm is exemplified by modifications with demonstrated roles in preventing miscoding, such as $\mathrm{xnm}^{5} \mathrm{U}$ in bacteria and $x \mathrm{~cm}^{5} \mathrm{~s}^{2} \mathrm{U}$ in eukaryotes (where $\mathrm{x}$ can be any of several different groups), as well as $s^{2} U$, which is responsible for decoding two-codon sets that end in purine ( $R$; NNR codons). Similarly, the presence of $\mathrm{mcm}^{5} \mathrm{U}_{34}$ (without thiolation) is known to improve the ability of tRNAs to read G-ending codons [12].

In vitro studies have also demonstrated that some tRNA modifications regulate translational accuracy by influencing 
the rejection of non-cognate codons at the ribosome A-site [13]. Other modifications at the anticodon loop serve to stabilize the binding energetics of the codon-anticodon pair, further supporting decoding fidelity by maintaining the translational reading frame $[8,14]$. Meanwhile, nucleoside modifications both in the anticodon stem loop (ASL) and in the structural core of the L-shaped structure of the tRNA contribute to the correct folding, stability and identity of tRNAs $[8,15-21]$.

Post-transcriptional modifications of tRNA have been identified in all species interrogated to date and cells commit a significant number of genes (more than 100 genes are associated with the biosynthesis of RNA modifications in yeast), as well as energy to this regulatory process. While tRNA-modifying enzymes and protein co-factors are highly conserved and appear to play important roles in regulating cellular responses to stress [22-24], most of these genes are non-essential under laboratory growth conditions $[6,25,26]$, and their functions remain poorly defined. Interestingly, recent classical genetics and GWAS studies have identified single-nucleotide polymorphisms (SNPs) in RNA-modifying enzymes genes in biological samples of individuals with cancer, neurodegenerative disease, diabetes, hearing loss and a range of metabolic syndromes [27], fuelling speculation that these enzymes may regulate specific subsets of diseasegenes. For example, mutations in the FTSJ1 methyltransferase, which modifies positions 32 and 34 of $\mathrm{tRNA}^{\text {Leu }}$, - ${ }^{\text {Phe }}$ and - Trp are found in patients with non-syndromic X-linked mental retardation [28]. The NSUN2 enzyme, which is responsible for inserting $\mathrm{m}^{5} \mathrm{C}$ at position 34 of $\mathrm{tRNA}^{\mathrm{Leu}}$, is highly expressed in colorectal and breast cancer [29], while the MELAS and the MERRF syndromes are associated with hypomodification of mitochondrial tRNAs, namely 5-taurinomethyluridine-tRNA ${ }^{\text {Leu }}$ (UUR; $\tau \mathrm{m}^{5} \mathrm{U}$ ) and 5-taurinomethyl2-thiouridine-tRNA ${ }^{\text {Lys }}\left(\tau \mathrm{m}^{5} \mathrm{~s}^{2} \mathrm{U}\right)[30,31]$. And deficiencies in 5-carbamoylmethyl and 5-methylcarboxymethyl side chains of tRNA wobble uridines $\left(\mathrm{ncm}^{5} \mathrm{U}\right.$ and $\mathrm{mcm}^{5} \mathrm{U}$, respectively) have been found to cause neurological and developmental defects in C. elegans [32].

While experimental evidence suggests that mutations in RNA-modifying enzymes may contribute to the pathogenesis of serious human diseases, a limited mechanistic understanding of how these enzymes control cellular phenotype under normal physiological conditions constrains our ability to rationally evaluate these genes as potential drug targets. Here we report the development of a yeast genetic screen to identify eukaryotic tRNA modifications required for proteostasis and whose absence results in increased protein aggregation. We used a library of 70 yeast strains, each containing a single-gene deletion of a tRNA-modifying enzyme and identified 5 genes whose absence correlated with significantly increased protein aggregation. Using homology-based spatial prediction and LC-MS/MS mapping of tRNA modifications, we found wobble uridine modifications $\mathrm{ncm}^{5} \mathrm{U}_{34}, \mathrm{mcm}^{5} \mathrm{U}_{34}$ and $\mathrm{mcm}^{5} \mathrm{~s}^{2} \mathrm{U}_{34}$ to be critical for protein folding and solubility. Computational analysis of the protein aggresomes of selected yeast mutants confirmed that the absence of these modifications correlated with increased rates of amino acid misincorporation at the codons decoded by hypomodified
tRNAs. Together, these findings suggest a role of tRNA modifications in proteostasis and identify the yeast tRNA modifications that maintain the protein synthesis rate and accuracy.

\section{Materials and Methods}

\section{Yeast library construction and growth characteristics}

All S. cerevisiae strains used derived from BY4743 (MATa/a; his $3 \Delta 1 /$ his $3 \Delta 1$; leu $2 \Delta 0 /$ leu $2 \Delta 0$; LYS2/lys $2 \Delta 0$; met $15 \Delta 0$ / MET15; ura3 $\Delta 0 /$ ura3 $\Delta 0$ ), here referred to as 'wild-type' (WT). To produce mutant strains, each gene of interest was replaced by the KanMX4 cassette (EUROSCARF; 33) (Table $\mathrm{S} 1$ ). Yeast strains were grown at $30^{\circ} \mathrm{C}$ in YPD medium (glucose: $2 \%(\mathrm{w} / \mathrm{v})$, yeast extract: $0.5 \%(\mathrm{w} / \mathrm{v})$, and peptone: $1 \%(\mathrm{w} /$ v) (Formedium) and minimal medium without histidine (MM-His; glucose: $2 \%(\mathrm{w} / \mathrm{v})$, yeast nitrogen base without amino acids: $0.67 \%(\mathrm{w} / \mathrm{v})$ plus each one of the required amino acids $(100 \mu \mathrm{g} / \mathrm{ml}))$ (Formedium).

Strains were transformed using a modified LiAc/SS Carrier DNA/PEG method [34]. Briefly, cells were grown overnight in YPD at $30^{\circ} \mathrm{C}$, with $180 \mathrm{rpm}$ shaking, until an $\mathrm{OD}_{600}$ of 0.4-0.5. Cells were then centrifuged (5000 rpm; 1 minute) and the supernatant was discarded. Transformation reagents and $3 \mu \mathrm{g}$ of DNA (GFP-His fusion cassette amplified from the plasmid pKT128 (pFA6a-GFP(S65T)-His3MX6)) were added to the pellet and incubated at $42^{\circ} \mathrm{C}$ for 40 minutes. Cells were then centrifuged at maximum speed for 1 minute, the transformation mixture (supernatant) was discarded, and pellets were carefully resuspended in $200 \mu \mathrm{L}$ of sterile milliQ water. Each cell suspension was plated on selective medium plates (MM-His) and incubated at $30^{\circ} \mathrm{C}$ until isolated transformant colonies were visible (2-4 days). Insertion of the fusion cassette by homologous recombination was confirmed by colony PCR for 3 biological replicates (clones).

Mutant strains were characterized by measuring growth rates as follows: (1) first, fresh medium (MM-His) was inoculated from a pre-culture and allowed to grow to an initial $\mathrm{OD}_{600}$ of 0.01 at $30^{\circ} \mathrm{C}, 180 \mathrm{rpm}$, until late stationary phase; (2) next, aliquots were removed from the culture and $\mathrm{OD}_{600}$ was measured using a Microplate Reader (BioRad) at multiple timepoints during growth; (3) finally, time-dependent $\mathrm{OD}_{600}$ values were plotted.

\section{In vivo detection of protein aggregates by fluorescence microscopy}

Yeast cells were harvested from $2 \mathrm{~mL}$ culture allowed to grow to an initial $\mathrm{OD}_{600}$ of 0.01 at $30^{\circ} \mathrm{C}, 180 \mathrm{rpm}$, until early logarithmic phase, and fixed for visualization using an Axio Imager.Z1 (Zeiss) epifluorescence upright microscope, a 63X oil-immersion objective and 38 HE GFP and Brightfield filters. Images were captured using AxionVision Software (Zeiss). The images were accumulated in one representative focal plane and subsequently processed and analysed using ImageJ software (http://rsb.info.nih.gov/ij). Intracellular fluorescent foci were confirmed and quantified manually, on average 500 cells of 3 clones per strain were analysed. 


\section{Total RNA extraction and tRNA purification}

For total RNA extraction cells were harvested from 250-mL cultures allowed to grow until logarithmic phase $\left(\mathrm{OD}_{600}\right.$ of 1-1.5). Cell pellets were washed 3 times with ice-cold phosphate-buffered saline and frozen at $-80^{\circ} \mathrm{C}$. Cells were resuspended in phenol-chloroform $(5: 1 v / v)$ at $\mathrm{pH} 4.7$ (phenol volume $=$ culture volume $\left.\times\left[\mathrm{OD}_{600} / 25\right]\right)$, and equal volume of TES buffer (10 mM Tris pH 7.5, $10 \mathrm{mM}$ EDTA, 0.5\% SDS). Cell suspensions were vigorously shaken for $30 \mathrm{sec}$ and incubated at $65^{\circ} \mathrm{C}$ for $1 \mathrm{~h}$ with agitation every $10 \mathrm{~min}$. RNAcontaining aqueous phase was separated from the phenolic phase by centrifugation at $7500 \mathrm{rpm}$ for $30 \mathrm{~min}$ at $4^{\circ} \mathrm{C}$, and re-extracted with the same volume of fresh phenol at $7500 \mathrm{rpm}$ for $20 \mathrm{~min}$ at $4^{\circ} \mathrm{C}$. Aqueous phases were once again re-extracted with the equivalent volume of chloroformisoamyl alcohol $(24: 1 \mathrm{v} / \mathrm{v})$ by centrifugation at $7500 \mathrm{rpm}$ for $20 \mathrm{~min}$ at $4^{\circ} \mathrm{C}$. RNA was precipitated overnight at $-30^{\circ} \mathrm{C}$ with 3 volumes of pure ethanol and 0.1 volumes of $3 \mathrm{M}$ sodium acetate at $\mathrm{pH}$ 5.2. RNA was harvested by centrifugation at $7200 \mathrm{rpm}$ for $30 \mathrm{~min}$ at $4^{\circ} \mathrm{C}$ and resuspended in $1 \mathrm{~mL}$ of $0.1 \mathrm{M}$ sodium acetate $\mathrm{pH}$ 4.5. tRNA was isolated on a $40-\mathrm{mL}$ DEAE-cellulose column equilibrated with RNA resuspension buffer, as previously described [35]. Briefly, samples were washed with 2.5 volumes of $0.1 \mathrm{M}$ sodium acetate ( $\mathrm{pH} 4.5) /$ $0.3 \mathrm{M}$ sodium chloride, and tRNA was eluted with 2 volumes of $0.1 \mathrm{M}$ sodium acetate ( $\mathrm{pH} 4.5$ )/ $1 \mathrm{M}$ sodium chloride. tRNA was precipitated with 2.5 volumes of $100 \%$ ethanol overnight at $-30^{\circ} \mathrm{C}$, harvested by centrifugation and resuspended in milli-Q (mQ) water and stored at $-80^{\circ} \mathrm{C}$. tRNA content was subsequently verified at room temperature by electrophoresis using $15 \%$ polyacrylamide- $8 \mathrm{M}$ urea gels, buffered with TBE.

\section{Isolation of tRNA by HPLC}

Purified tRNA was analysed using Agilent RNA 6000 pico assay. HPLC-based purification of tRNA was carried out using an Agilent 1100 HPLC series coupled to an Agilent Bio SEC-3 $300 \AA$ column (300 mm length $x 7.8 \mathrm{~mm}$ inner diameter) with a temperature-controlled column compartment kept at $60^{\circ} \mathrm{C}$ and a $100-\mathrm{mM}$ ammonium acetate aqueous phase (isocratic gradient) at a flow rate of $1 \mathrm{~mL} / \mathrm{min}$ for 15 min. tRNA peaks were detected by measuring absorbance at $260 \mathrm{~nm}$ and collected with a fraction collector using predetermined retention times. Fractions were concentrated by SpeedVac and rehydrated with milliQ water.

\section{Quantitative analysis of tRNA modifications by LC-MS/MS}

Modified ribonucleosides in tRNA were identified and quantified, as reported previously [36]. Briefly, isolated tRNA were enzymatically hydrolysed and dephosphorylated by incubating for $2 \mathrm{~h}$ at $37^{\circ} \mathrm{C}$ with coformycin $(50 \mu \mathrm{g} / \mathrm{mL})$, THU $(0.3 \mathrm{mg} / \mathrm{mL}), \mathrm{MgCl} 2(0.5 \mathrm{mM})$, Tris $\mathrm{pH} 8.0(0.2 \mathrm{M})$, alkaline phosphatase $(0.05 \mathrm{U} / \mu \mathrm{L})$, PDE1 $(0.005 \mathrm{U} / \mu \mathrm{L})$, BHT $(0.3 \mathrm{mM})$ and Benzonase $(0.03 \mathrm{U} / \mu \mathrm{L})$. Enzymes were removed from digested ribonucleosides by ultrafiltration using a $10-\mathrm{kDa}$ membrane (12,000 x g, 4-10 $\mathrm{min}$ ), and ribonucleosides were subsequently spiked with $\left[{ }^{15} \mathrm{~N}\right]_{5}-\mathrm{dA}$ as an internal control.
Purified ribonucleoside standards were used to optimize mass spectrometry detection and fragmentation parameters. 10 pmol of each ribonucleoside standard was injected by ultra-high performance liquid chromatography (UPLC) coupled to a triple quadrupole (QQQ) mass spectrometer with $5 \mathrm{mM}$ ammonium acetate as the solvent. The UPLC setup involved a Synergy $2.5-\mu \mathrm{m}$ Fusion - RP 100- $\AA$ (100 x $2 \mathrm{~mm}$ ) column operated with a mobile phase of 0 to $80 \%$ acetonitrile $(\mathrm{ACN})$ in $5 \mathrm{mM}$ ammonium acetate at a flow rate of $350 \mu \mathrm{L} / \mathrm{min}$ and $35^{\circ} \mathrm{C}$. The HPLC column was coupled to an Agilent 6430 QQQ mass spectrometer with an electrospray ionization (ESI) source operated in positive ion mode with the following parameters: gas temperature $=350^{\circ} \mathrm{C}$; gas flow $=10.0 \mathrm{~L} / \mathrm{min}$; nebulizer $=40.0 \mathrm{psi}$; capillary current $=5549 \mathrm{nA}$. Ribonucleoside standards were identified by HPLC retention time and collision-induced dissociation (CID) fragmentation pattern.

Digested ribonucleosides were analysed by UPLC-MS/MS, and modifications were quantified by dynamic multiple reaction monitoring (MRM) using molecular transitions previously calculated from ribonucleoside standards. After background subtraction, the signal intensity of each ribonucleoside was normalized against signal intensity of the $\left[{ }^{15} \mathrm{~N}\right]_{5^{-}}$ dA internal standard, permitting adjustments for day-to-day fluctuations in MS sensitivity. Signal intensity for each ribonucleoside was also normalized by dividing the raw peak area for the ribonucleoside by the sum of the UV absorbance peak areas for the four canonical ribonucleosides, in order to adjust for variations in total tRNA content in each sample. For each sample, measurements were acquired across 3 biological replicates (clonal isolates) using 3 technical replicates, and statistical significance was determined using a Student's $t$-test. Quantified nucleoside modification data for each mutant strain was subsequently transformed to $\log _{2}$ (fold change) ratios relative to WT. Hierarchical clustering analysis was performed using Cluster 3.0: average linkage algorithm based on the distance between each data set measured using Euclidean distance, with the heat map representations produced using Java TreeView.

\section{tRNA isoacceptor quantification by four-leaf clover qRT-PCR}

Hypomodified tRNA was quantified using a polymerase chain reaction- (PCR-) based method described in Honda et al., 2015 [37]. Sequences of adapters and primers for four-leaf clover reverse transcriptase PCR (FL-qRT-PCR) are shown in Table S2. Briefly, total RNA was subjected to deacylation treatment (incubation in $20 \mathrm{mM}$ Tris- $\mathrm{HCl} \mathrm{pH} 9.0$ at $37^{\circ} \mathrm{C}$ for $40 \mathrm{~min}$ ), followed by annealing and ligation with a DNA/ RNA-hybrid stem-loop adapter using T4 RNA ligase 2 (New England Biolabs), which specifically ligates the adapter to mature tRNA. Ligated tRNAs were specifically amplified and quantified by TaqMan qRT-PCR with an Applied Biosystems 7500 Real-Time PCR System, using a standard program of $95^{\circ}$ $\mathrm{C}$ for $20 \mathrm{sec}$ followed by 40 cycles $\left(95^{\circ} \mathrm{C}\right.$ for $5 \mathrm{sec}, \mathrm{Tm}$ for $34 \mathrm{sec}$ ). All reactions were run in triplicate and the threshold cycles $(\mathrm{Ct})$ were determined. The amplified cDNA was developed by $10 \%$ native PAGE. For statistical evaluations of the 
determined $\mathrm{CP}$ and relative expression variations, data were analysed for significant differences by REST-MCS॰ using 3 independent clones with 3 technical replicates [38].

\section{Extraction of insoluble aggregates by differential centrifugation}

Insoluble protein fractions were isolated as described by Koplin et al., 2010, with a few modifications [39]. $20 \mathrm{OD}_{600}$ units of logarithmically growing cells cultivated in MM-His were harvested at $4000 \mathrm{rpm}$ for 10 minutes at $4^{\circ} \mathrm{C}$, and resulting cell pellets were washed with ice-cold phosphatebuffered saline (PBS) and frozen at $-80^{\circ} \mathrm{C}$. To prepare cell lysates, pellets were resuspended in $500 \mu \mathrm{L}$ of lysis buffer (20 mM Na-phosphate, pH 6.8, 10 mM DTT, 1 mM EDTA, 0.1\% $(v / v)$ Tween, $1 \mathrm{mM}$ PMSF, protease inhibitor cocktail (Roche), $3 \mathrm{mg} / \mathrm{mL}$ lyticase and $1.25 \mathrm{U} / \mathrm{mL}$ benzonase), and incubated for $30 \mathrm{~min}$ at $30^{\circ} \mathrm{C}$. Glass beads were used to disrupt yeast cells using a Precellys $\mathrm{s}^{\text {ti }} 24$ disrupter; 2 cycles of $25 \mathrm{sec}$ at $6500 \mathrm{rpm}$; samples were kept on ice between each cycle. Cell lysates were then centrifuged for $20 \mathrm{~min}$ at $200 \mathrm{x} \mathrm{g}$ at $4^{\circ} \mathrm{C}$ to isolate supernatant protein fractions. Following centrifugation, supernatant fractions were aspirated and adjusted to the same protein concentration $(5 \mathrm{mg} / \mathrm{mL}$ for protein gels) across samples. Following aspiration of the supernatant, membrane and aggregated proteins were isolated by centrifugation at $16,000 \mathrm{x} \mathrm{g}$ for $20 \mathrm{~min}$ at $4^{\circ} \mathrm{C}$. Following this round of centrifugation, resulting supernatant fractions were aspirated and membrane proteins were removed by resuspending aggregated proteins in $2 \% \mathrm{NP}-40$ (in $20 \mathrm{mM}$ Na-phosphate, $\mathrm{pH}$ 6.8, $1 \mathrm{mM}$ PMSF and protease inhibitor cocktail), disrupting the mixture by probe sonication (3 x $5 \mathrm{sec}$ at cycle 0.1 and amplitude 20\%), and centrifuging the mixture at $16,000 \mathrm{xg}$ for $20 \mathrm{~min}$ at $4^{\circ} \mathrm{C}$. This process was repeated twice, after which final insoluble protein fractions were washed with NP-40-deficient buffer (centrifuging the mixture at $16,000 \times \mathrm{g}$ for $20 \mathrm{~min}$ at $4^{\circ} \mathrm{C}$ ), solubilized in $50 \mu \mathrm{L}$ of Urea Buffer $(50 \mathrm{mM}$ Tris- $\mathrm{HCl}, \mathrm{pH} 7.5,6 \mathrm{M}$ urea and $5 \%$ SDS), boiled in $1 \mathrm{X}$ laemli sample buffer, separated by SDS-PAGE (14\%) and resolved by Coomassie staining.

For Mass spectrometry analysis, insoluble protein aggregates dissolved in NP-40-deficient buffer were precipitated with TCA $\left(100 \% w / v ; 1 \mathrm{vol}\right.$ of TCA to $4 \mathrm{vol}$ of protein sample) at $4^{\circ} \mathrm{C}$ for $30 \mathrm{~min}$. Precipitated protein was washed with $200 \mu \mathrm{L}$ ice cold acetone $(9: 1 v / v)$, and pellets were allowed to air dry at RT. Dried protein pellets were resuspended in $50 \mu \mathrm{L}$ of $8 \mathrm{M}$ urea before further processing for MS/MS analysis.

\section{Pan-proteome quantification of amino acid misincorporations}

Insoluble protein was reduced with $10 \mathrm{mM}$ DTT $\left(1 \mathrm{~h}\right.$ at $\left.56^{\circ} \mathrm{C}\right)$ and alkylated with $55 \mathrm{mM}$ iodoacetamide (1 h at RT in the dark). Protein was then digested overnight using modified trypsin (Thermo Scientific) at an enzyme/substrate ratio of 1:50 in $100 \mathrm{mM}$ ammonium acetate $(\mathrm{pH} 8)$ at $30^{\circ} \mathrm{C}$. Synthetic peptides mimicking amino acid misincorporations (designed in house) were added as internal controls to each sample (Table S3). Peptide mixtures were desalted using MicroSpin C18 columns
(The Nest Group, Inc) and analysed using an Orbitrap Fusion Lumos mass spectrometer (Thermo Scientific, San Jose, CA, USA) coupled to an Easy-nLC 1200 liquid chromatography system (Thermo Scientific (Proxeon), Odense, Denmark). Peptides were loaded directly onto the analytical column and separated by reverse-phase chromatography using a $50-\mathrm{cm}$ column with an inner diameter of $75 \mu \mathrm{m}$, packed with $2 \mu \mathrm{m}$ C18 particles (Thermo Scientific, San Jose, CA, USA). A 90-min chromatographic gradient using $0.1 \%$ formic acid in water (buffer A) and $0.1 \%$ formic acid in ACN (buffer B) was performed for each sample analysis at a flow rate of $300 \mathrm{~nL} / \mathrm{min}$ as follows: $0-79 \mathrm{~min}=$ start at $5 \%$ buffer B and gradually increase to $78 \%$ buffer B; 79-90 $\mathrm{min}=$ gradually decrease to $35 \%$ buffer $\mathrm{B}$. The column was washed for 10 min using $95 \%$ buffer B after each analytical gradient, and stored overnight at $95 \%$ buffer $\mathrm{A}$.

The Orbitrap was operated in data-dependent acquisition (DDA) mode and full MS scans (with 1 micro scan at resolution of 120,000) were used over a mass range of $350-1,500 \mathrm{~m} /$ z. Auto gain control (AGC) was set to 1E5 and dynamic exclusion to $50 \mathrm{sec}$. In each cycle of DDA analysis, ions that met defined detection criteria (peptide isotopic profiles, charge states between $2+$ and $5+$, ion count above 1E4) were selected for fragmentation at normalized collision energy of $28 \%$. Fragment ion spectra produced via higher-energy collision dissociation (HCD) were acquired using the following Orbitrap settings: $A G C=3 \mathrm{E} 4$, isolation window $=1.6 \mathrm{~m} / \mathrm{z}$, and maximum injection time $=80 \mathrm{~ms}$. All data were acquired and pre-filtered using Xcalibur (v3.0.63).

MS/MS raw data were analysed using PEAKS Studio (v.8.0, Bioinformatics Solutions Inc.) for peptide identification and label-free quantification: (1) Samples were searched against a $S$. cerevisiae database sourced from the Saccharomyces Genome Database (version of July 2017), which included a comprehensive list of decoy entries and common contaminants; (2) trypsin was designated as the digestive enzyme and a maximum of three missed cleavages were allowed; (3) carbamidomethylation was set as a fixed modification and oxidation (M) was set as a variable modification; (4) searches were performed using mass tolerances of $7 \mathrm{ppm}$ for MS scans and $20 \mathrm{mmu}$ for MS/MS scans; and (5) resulting search results were filtered for FDR $\leq 1 \%$. Differential analysis of protein abundance was analysed with DAPAR and ProStaR packages of R. Samples were normalized with the quantile centring method, setting the value of quantile to $0.15 \%$, assuming that the signal/noise ratio is roughly the same in all the samples. Missing values in the 3 biological replicates of a mutated strain were imputed to value 1 , whereas missing values in 1 or 2 biological replicates were imputed to the minimum value of the respective sample. Upregulated aggregated proteins (2-logFC) were identified using a Limma statistical test, with FDR $\leq 5 \%$ and $\mathrm{p}$ value $\leq 0.05$ after calibration (pounds).

Amino acid misincorporations were identified using the SPIDER algorithm of PEAKS Studio (v.8.5, Bioinformatics Solutions Inc.), as described above with several changes: (1) raw data was searched against the S. cerevisiae database; (2) trypsin was chosen as the digestive enzyme with a maximum of three allowable missed cleavages; (3) carbamidomethylation 
was set as a fixed modification, whereas oxidation (M), Asn $\rightarrow$ Lys substitution, Asp $\rightarrow$ Glu substitution, His $\rightarrow$ Gln substitution, Phe $\rightarrow$ Leu substitution, and Ser $\rightarrow$ Arg substitution were set as variable modifications; (4) searches were performed using mass tolerances of $7 \mathrm{ppm}$ for MS scans and $20 \mathrm{mmu}$ for MS/MS scans; (5) additional search parameters were analysed to identify common and unspecified posttranscriptional modifications (PTM); and (6) resulting search results were filtered for FDR $\leq 1 \%$. Amino acid misincorporations were validated bioinformatically by programming misincorporations into a new $S$. cerevisiae protein database and re-searching raw data with PEAKS without designating amino acid substitutions as variable modifications. Amino acid misincorporations were considered valid only if bioinformatically mutated proteins (encoding mutant peptide sequences) were accurately identified in the second database search at $\leq$ $1 \%$ FDR.

\section{Results}

\section{Deletion of tRNA modification genes induces protein aggregation}

$S$. cerevisiae encodes 73 genes involved in tRNA modification, of which 21 have been shown to be essential or affect growth.
We engineered a diploid yeast gene knockout library to characterize protein aggregation phenotypes of 50 homozygotic nonessential genes (two mutants - Dus $1 \Delta$ and $\operatorname{Pcc} 1 \Delta$ failed quality control), as well as 17 heterozygotic (single-copy deletions) essential genes or genes that confer significantly retarded growth (Tables S1 and S4). Our knockout library included the catalytic subunits of tRNA-modifying enzymes, as well as other enzymes that indirectly affect tRNA modifications - such as Kti12, a kinase required for Elongator function in vivo.

We developed a microwell plate-based fluorescence aggregometry platform to assess how shifts in endogenous tRNA modification profiles affect protein aggregation phenotypes in yeast. This assay employs a protein aggregate sensor that we engineered by fusing Hsp104 and GFP open reading frames under control of the yeast Hsp104 promoter (Fig. 1A). As Hsp104 is a disaggregase known to recognize and bind aggregated proteins, our Hsp104-GFP construct permitted rapid visualization and quantification of protein aggregates by epifluorescence and confocal microscopy [40,41]. This sensor was transformed into each strain in our KO library, and resulting cells were screened by epifluorescence microscopy (Fig. 1B). Cells containing highly soluble protein content had GFP fluorescence homogenously distributed throughout the
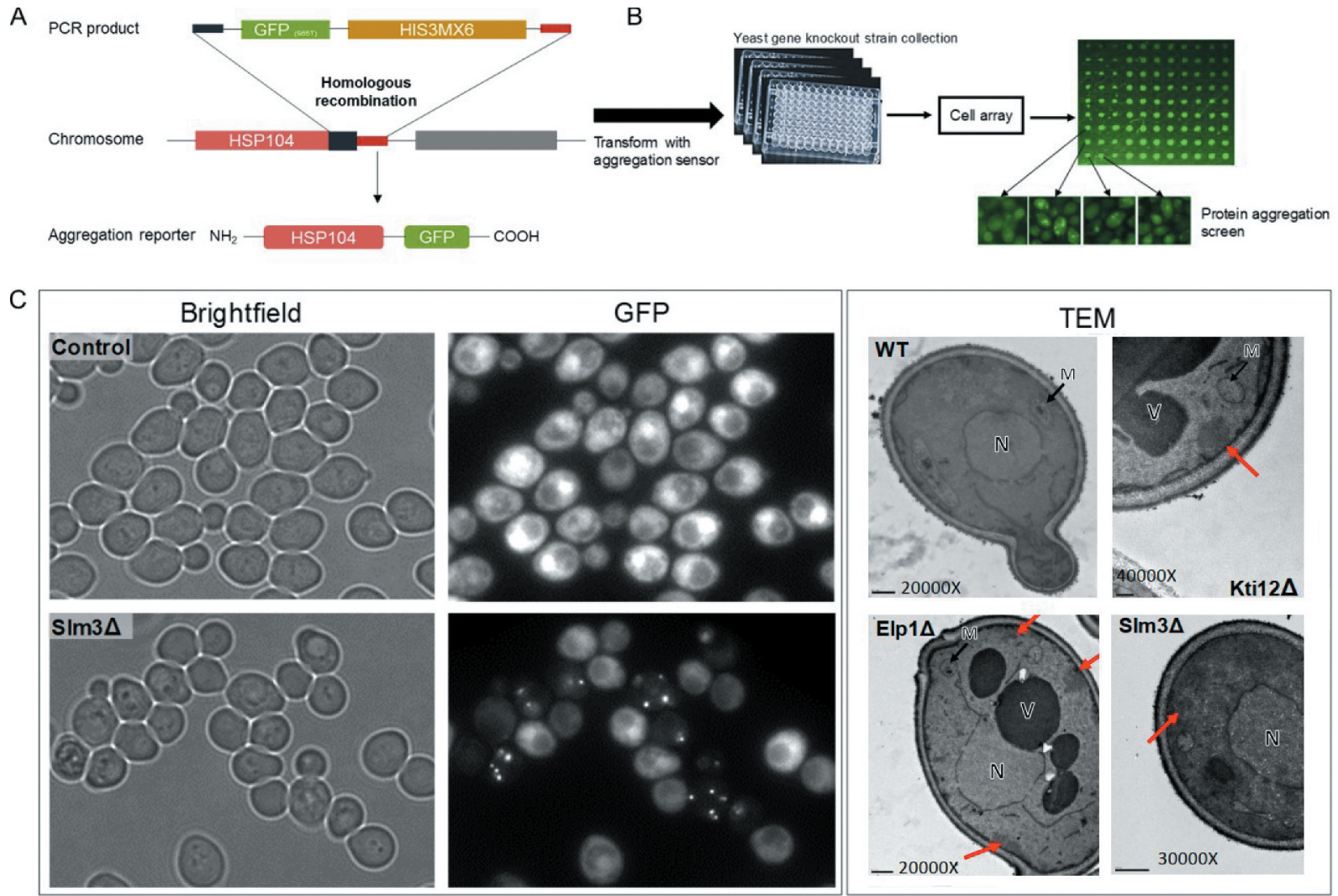

Figure 1. Screen to identify RNA-modifying enzyme KOs that induce protein aggregation. A. Engineering scheme for the construction of a fluorescent molecular sensor of protein aggregation. PCR products containing a GFP tag and a selectable marker gene (plasmid pKT128 - pFA6a-GFP(S65T)-His3MX6) were fused in-frame to the C-terminal coding region of the Hsp104 gene through homologous recombination, yielding an Hsp104-GFP fusion protein. Adapted from [72]. B. Diagram outlining experimental strategy of screening our engineered fluorescent library to identify RNA-modifying enzyme KOs with protein aggregate phenotypes. C. Left box: WT (BY4743) and the mutant $\operatorname{SIm} 3 \Delta$ yeast strains expressing the Hsp104-GFP reporter protein were collected in mid-exponential growth phase and observed by fluorescence microscopy (60x objective). Cells harbouring deletion in the SIm3 gene showed localized Hsp104-GFP fluorescence, indicating the presence of protein aggregates. Right box: Ultrastructure of WT and Elp1 $\Delta$, Kti12 $\Delta$ and SIm3 3 mutant yeast cells captured using Transmission Electron Microscopy (TEM) of Epon 812 resin embedded cells. Red arrows indicate regions with increased electron-dense protein aggregate content. N: nucleus; M: mitochondrion; V: vacuole. 
cytoplasm, while cells containing protein aggregates displayed highly concentrated fluorescent loci (Fig. 1C left). We further analysed these strains by transmission electron microscopy (TEM) to distinguish protein aggregates from organelle membranes also present in the cytoplasm (Fig. 1C right, red arrows). TEM analysis of WT cells did not show the electrondense aggregates observed in the mutant strains (Fig. 1C).

Relative to the WT, cells with deletion of Elp1, Elp3, Kti12, Slm3 and Trm9 showed significantly increased localization of Hsp104-GFP in aggregate foci (Fig. 2). Notably, these five strains harbour deletions in genes encoding enzymes that are involved in modifying tRNA wobble uridines. Trm9 is a wobble uridine methyltransferase that catalyzes the esterification of modified uridine nucleosides, producing $\mathrm{mcm}^{5} \mathrm{U}_{34}$ and $\mathrm{mcm}^{5} \mathrm{~s}^{2} \mathrm{U}_{34}$ in specific tRNAs [42]. Elp1 and Elp3 are subunits in the Elongator complex, which is required for the formation of the $\mathrm{cm}^{5} \mathrm{U}$ side chain in wobble uridines [43]. Kti12 is important for the phosphorylation of Elp1 and is also required for the formation of $\mathrm{mcm}^{5}$ and $\mathrm{ncm}^{5}$ side chains at wobble uridines [43]. $\operatorname{Slm} 3$ is a known mitochondrial tRNA-specific 2-thiouridylase 1 (Mtu1) responsible for the formation of the $s^{2}$ group in $\mathrm{cmnm}^{5} \mathrm{~s}^{2} \mathrm{U}_{34}$-containing tRNA species (only in mitochondrial tRNAs) [31]. Of note, the respective formation of $\mathrm{mcm}^{5}$ and $\mathrm{s}^{2}$ groups occurs through independent biosynthetic processes.

We next examined whether $\mathrm{KO}$ strains with significantly increased protein aggregation also exhibited slower growth and/or loss of cell viability (Fig. 3), and observed a strong decrease in the cells growth relative to WT in the Elp1 $\Delta$, Kti12 $\Delta$ and Trm9 $\Delta$ strains, which agreed with previous reports [44-46]. However, the other $\mathrm{KO}$ strains also exhibited slower growth rates relative to WT (Fig. 3A, B). Cell viability appears not to be significantly altered (Fig. 3C). In other words, cytotoxic protein aggregates result from the disruption of certain tRNA-modification genes, repress growth rate and promote cell death.

\section{Deleting tRNA modification genes alters tRNA stability and modification levels}

We used LC-MS/MS to quantitatively profile variations in the global abundance of tRNA modifications across 4 of the 5 tRNAmodifying enzyme mutants selected by fluorescence aggregometry (Fig. 4A). Elp3 $\Delta$ was not analysed as it was already described in the literature $[47,48]$. Analysis by LC-MS/MS revealed decreased $\mathrm{ncm}^{5} \mathrm{U}$ and $\mathrm{ncm}^{5} \mathrm{Um}$ levels to limits close to detection in Elp1 $\Delta$ and Kti12 $\Delta$, while $\mathrm{mcm}^{5} \mathrm{U}$ and $\mathrm{mcm}^{5} \mathrm{~s}^{2} \mathrm{U}$ levels were also significantly reduced in Trm $9 \Delta$, Elp $1 \Delta$ and Kti12 $\Delta$ (Fig.s $4 \mathrm{~B}$ and S2; Table S5). Meanwhile, the Slm3 thiolase KO showed lower levels of $\mathrm{mcm}^{5} \mathrm{~s}^{2} \mathrm{U}$ and increased levels of $\mathrm{mcm}^{5} \mathrm{U}$, which is consistent with the absence of uridine thiolation (Fig.s $4 \mathrm{~B}$ and S2; Table S5). We also measured statistically significant decrease in yW levels across Kti12 $\Delta$, Trm $9 \Delta$ and $\operatorname{Sim} 3 \Delta$. These findings confirmed that inactivation or deletion of tRNA-modifying enzymes alters the levels of compensatory tRNA modifications.

Differences in detectable tRNA modification levels can result from either differential enzymatic activity or differences in tRNA isoacceptor abundance. To distinguish the variables contributing to variation in tRNA modification levels we also used FT-qRT-PCR to measure the abundance of the hypomodified tRNAs in the tRNA-modifying enzyme mutants [37]. Our analysis suggests that levels of tRNAs modified by Trm9, respectively, are not significantly affected by tRNA hypomodification (Fig. 4C), which agrees with previous studies of the Trm9 $\Delta$ and the Tucl $\Delta$ thiolase [12]. In contrast, we observed significantly reduced tRNA levels in both Elp1s (tRNA ${ }_{\text {ncm5UGC, }}^{\text {Ala }}$ $\mathrm{tRNA}^{\text {Thr }}{ }_{\text {ncm5UGU }}$ and tRNA $^{\text {Arg }}{ }_{\text {mom5UCU) }}$ and Ktil2 $\Delta$
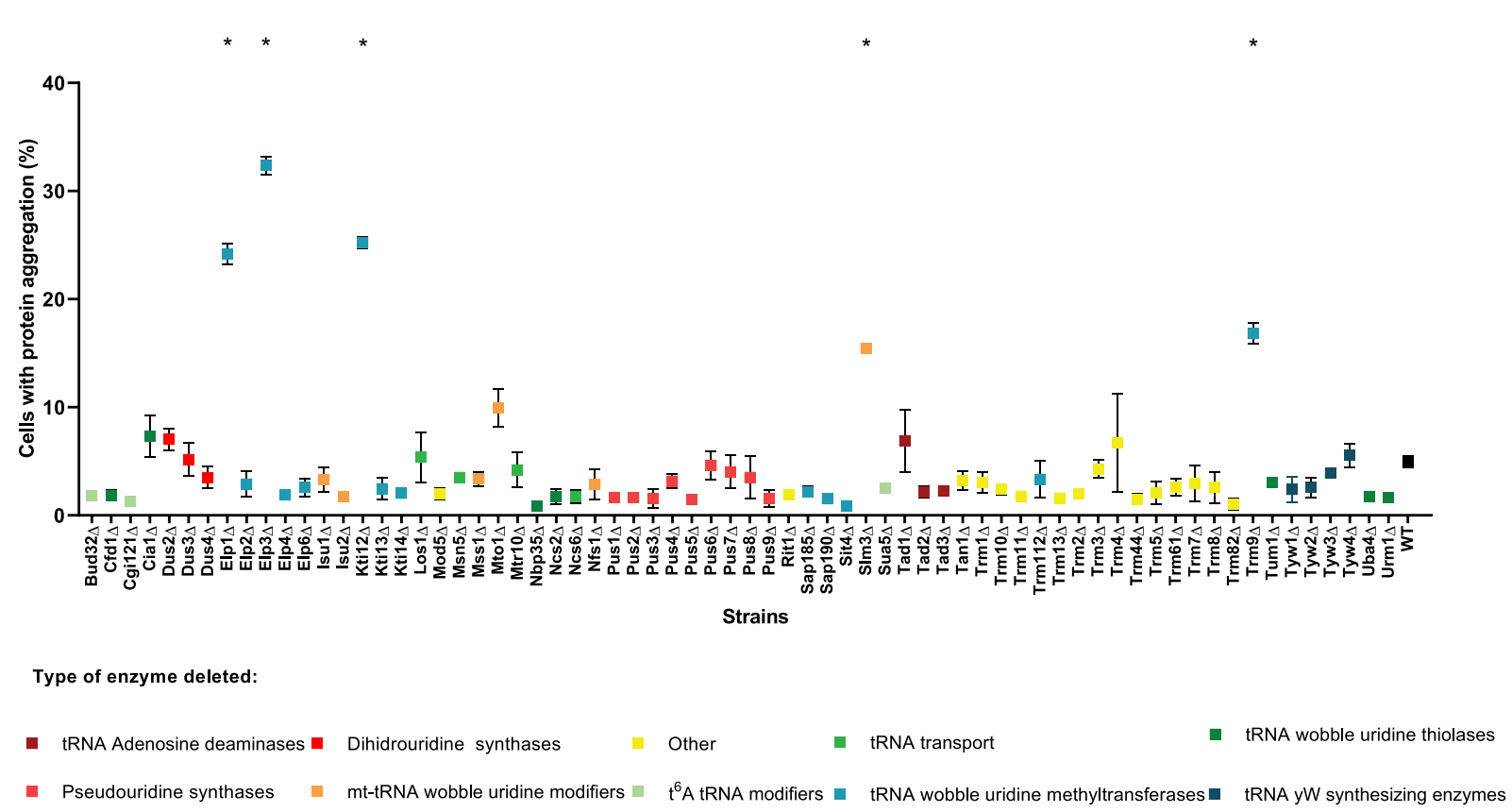

Figure 2. Protein aggregate phenotypes vary amongst tRNA-modifying enzyme KO strains. Data shows the mean \pm SEM of the percentage of cells containing localized Hsp104-GFP fluorescent foci. For each KO strain, data represents triplicates of 3 independent clones ${ }^{*} p<0.001$ One way ANOVA post Dunnett's multiple comparison test and $\mathrm{Cl} 95 \%$ relative to WT). 
A

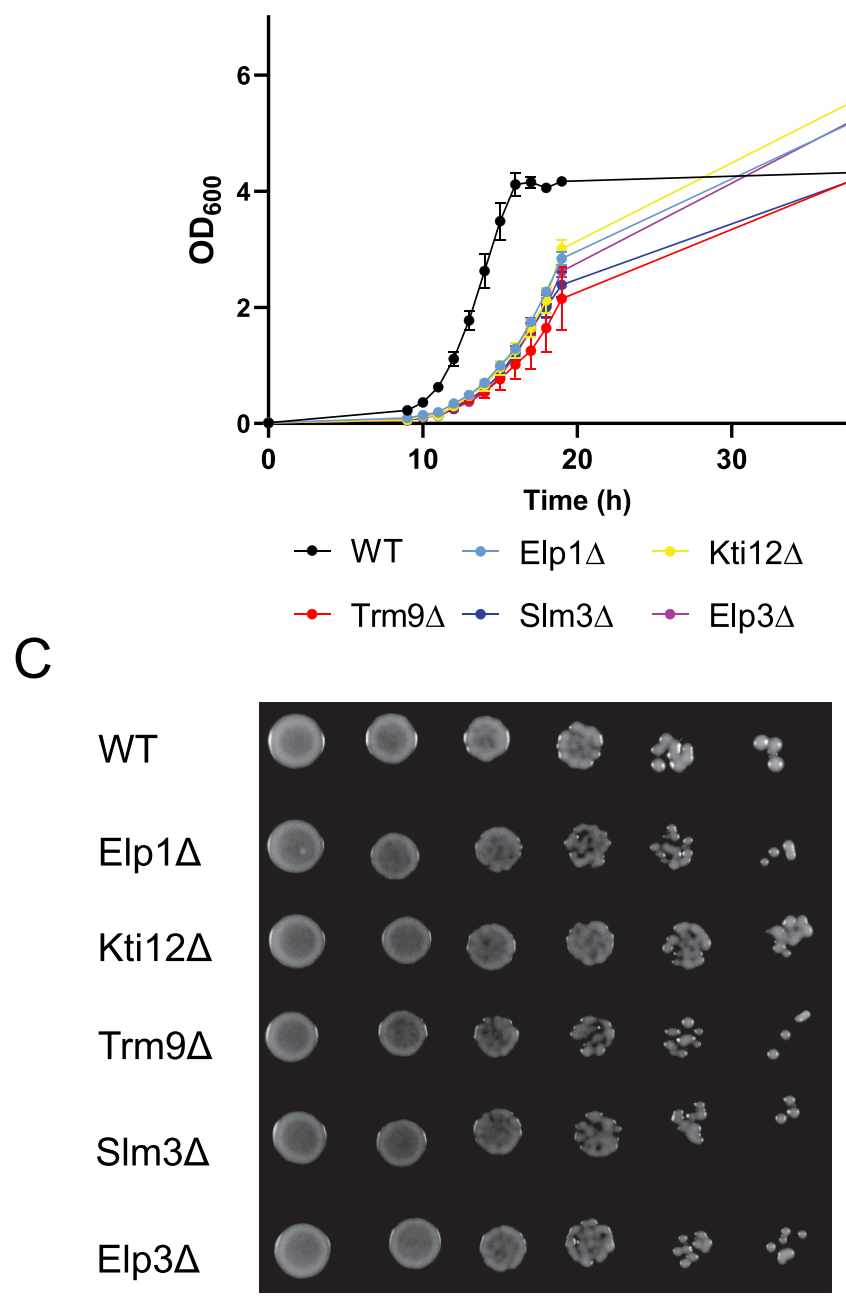

B
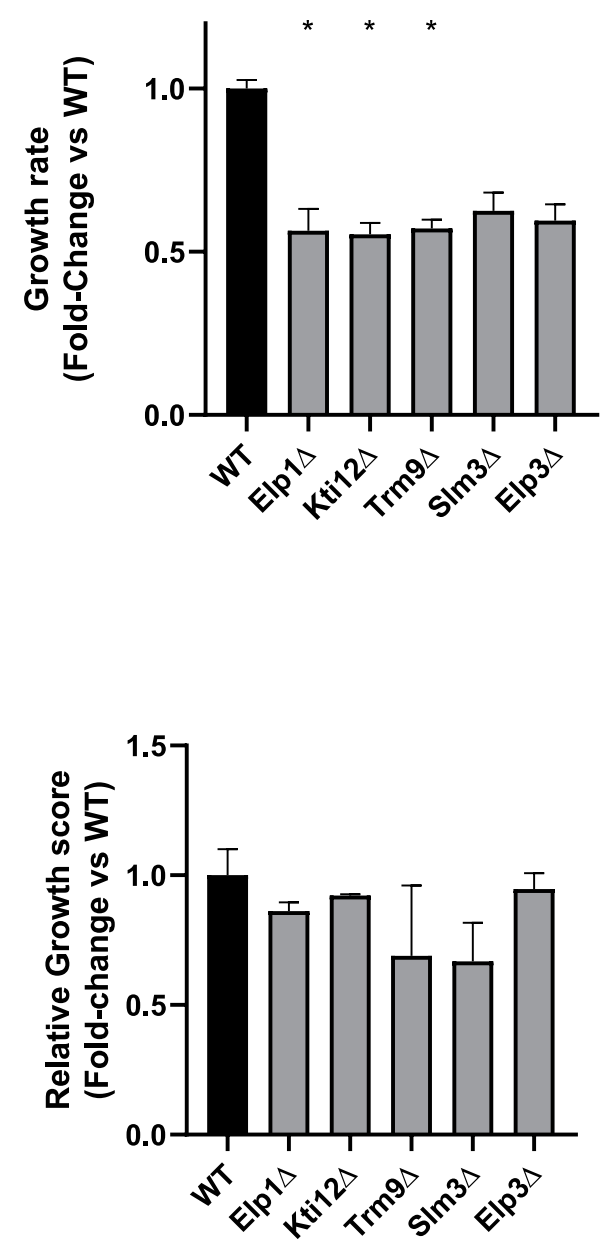

Figure 3. Physiological characteristics of selected RNA-modifying yeast mutants. A. Yeast cultures were inoculated at an initial $\mathrm{OD}_{600}$ of 0.01 and grown to stationary phase in selective medium (MM-His) at $30^{\circ} \mathrm{C}$ and $180 \mathrm{rpm}$. Optical density was measured at timepoints along the growth curve. B. Relative growth rate of mutant strains was determined by normalizing the exponential growth rate of each KO strain to the exponential growth rate of WT cells. Data represents the mean \pm SD of duplicate measurements for each of three independent clones $\left({ }^{*} p<0.05\right.$ Kruskal-Wallis test post Dunn's multiple comparisons test with Benjamini-Hochberg adjustment and $\mathrm{Cl}$ of $95 \%$, relative to WT). C. Colony survival of selected mutant strains compared to WT strain. Yeast cells grown in stationary phase were diluted to an initial $\mathrm{OD}_{600}$ and 6 fivehold serial dilutions onto $\mathrm{MM}$-His plates were spotted. Their colony-forming abilities were then analysed after 2 days of incubation at $30^{\circ} \mathrm{C}$. The relative growth score represents a ratio between growth of each KO strain normalized against growth of the WT strain. Data represent mean \pm SD of three independent clones.

(tRNA $^{\text {Gln }}{ }_{\text {mcm5s2UUG }}, t_{R N A}{ }^{\text {Glu }}{ }_{\text {mcm5s2UUC, }}, \mathrm{tRNA}^{\text {Thr }}{ }_{\text {ncm5UGU }}$ and tRNA $^{\text {Arg }}{ }_{\text {mom5UCU), suggesting that Elp1 and Kti12 mediated }}$ tRNA modifications either directly or indirectly regulate tRNA stability (Fig. 4C). These findings indicate that tRNA modifications are important not only for proteostasis but also for the abundance of certain tRNA isoacceptors.

\section{Codon biases exponentiate protein aggregation in yeast strains harbouring tRNA mutations}

We next sought to investigate the mechanistic link between altered tRNA modification, isoacceptor abundance and increased protein aggregation observed in tRNA-modifying enzyme KOs. Using differential density centrifugation, we isolated aggregated protein fractions from exponentially growing WT, Trm9 4 , Elp $1 \Delta, \operatorname{Kti} 12 \Delta, \operatorname{Slm} 3 \Delta$ and Elp3 $\Delta$ cells. We used SDS-PAGE to visualize differences between total and insoluble protein fractions across mutants (Fig. 5A and Figure S3), which confirmed the results of fluorescence aggregometry.

We then used LC-MS/MS to identify and quantify insoluble proteins from the mutants $\operatorname{Elp} 1 \Delta, \operatorname{Slm} 3 \Delta$ and $\operatorname{Trm} 9 \Delta$. Relative to WT, we measured the significant upregulation $\left(\log _{2} \mathrm{FC}>2\right)$ of 283,309 and 355 proteins in Elp $1 \Delta, \operatorname{Slm} 3 \Delta$ and $\operatorname{Trm} 9 \Delta$, respectively (Tables S7-S9). Among the upregulated insoluble proteins identified 144 proteins were common to all mutant strains (Fig. 5B).

Gene ontology (GO) analysis revealed that aggregated proteins in Trm9 $\Delta$ are involved in metabolic processes and their regulation, DNA repair, protein modification and regulation of gene expression (Fig. 5C). Aggregates in the Elp1 $\Delta$ strain were enriched in functions related to positive regulation of 

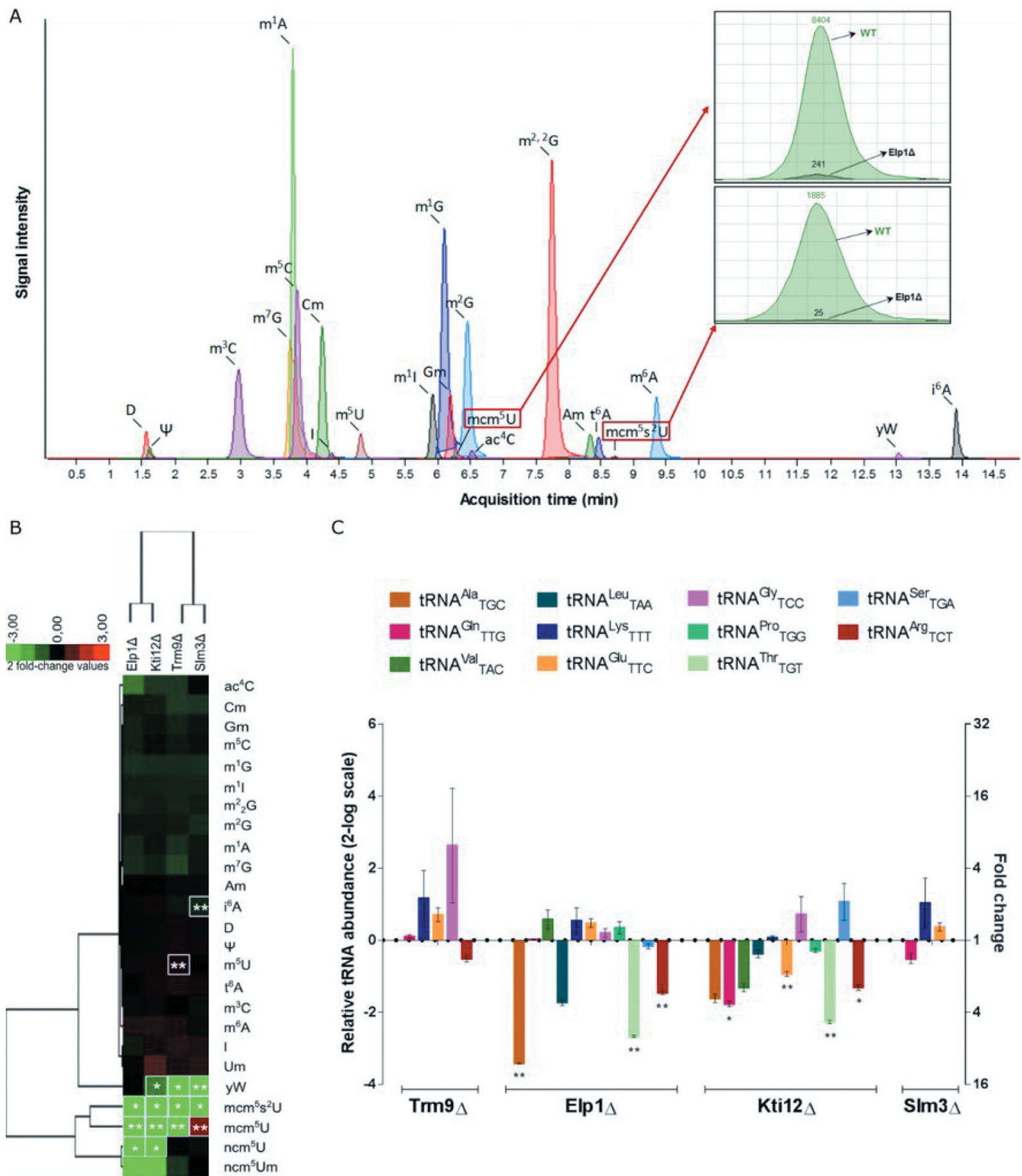

C
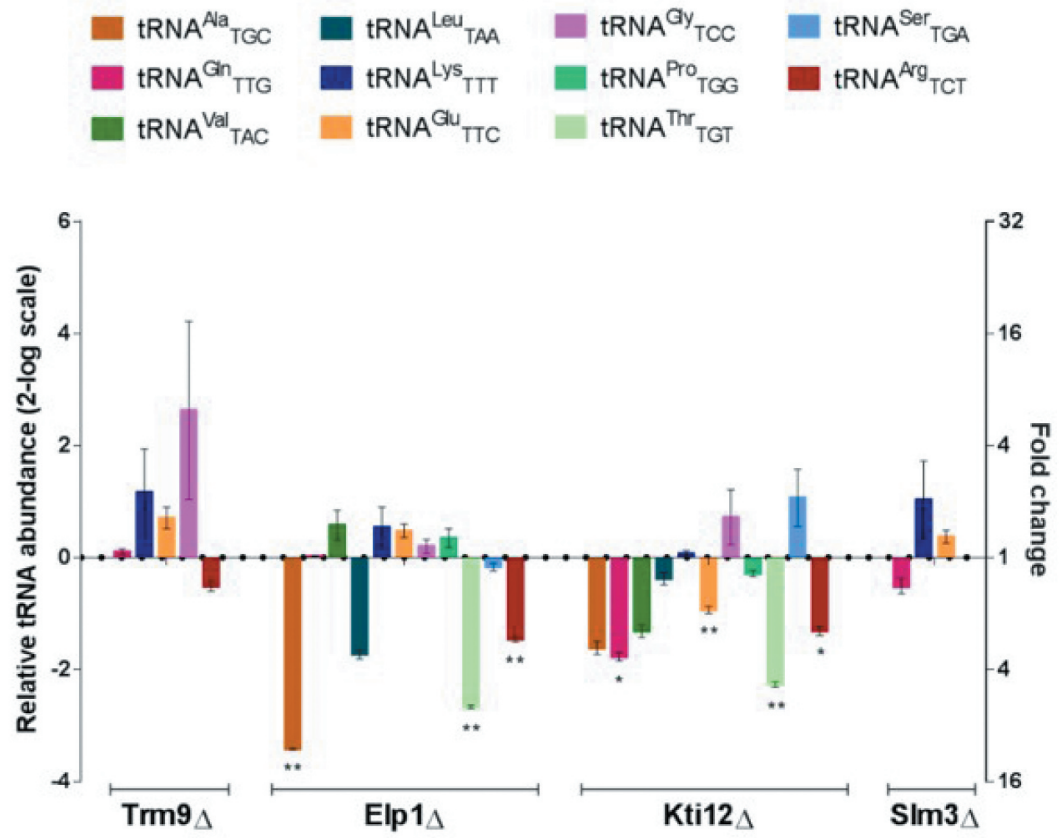

Figure 4. Knocking out tRNA-modifying enzymes affects steady-state tRNA modification profiles and abundance. A. Total ion chromatogram of LC-MS/MS analysis of modified tRNA ribonucleosides (WT). Chromatograms for $\mathrm{mcm}^{5} \mathrm{U}$ and $\mathrm{mcm}^{5} \mathrm{~s}^{2} \mathrm{U}$ comparing WT with Elp1 $\Delta$ strains confirm reduced modification levels in the Elp1 $\Delta$ strain. Data for other strains are shown in supplementary Figure S2. B. Hierarchical cluster of the relative abundance of tRNA ribonucleoside modifications in selected mutant strains. TRNA modifications were identified and quantified by mass-spectrometry. Log-transformed fold-change values for mutants were normalized against WT, and the resulting ratios were subjected to hierarchical cluster analysis. Data represent mean of triplicates of three independent clones (** $\mathrm{p}<0.01, * \mathrm{p}<0.05$, Student's t-test with CI 95\% relative to WT). Red: fold increase; Green: fold decrease; according to the scale in the top-left colour bar. C. Quantification of wobble modified tRNAs in selected tRNAmod KO strains. The plot illustrates the abundance of each tRNA in mutant strains relative to quantified levels of the respective tRNAs in WT. Data was obtained using the four-leaf clover qRT-PCR method [37], and represents relative tRNA abundance \pm SD in a 2-log scale of triplicates of 3 independent clones (calculated with REST software [38]).

nuclear division and cellular processes, and $\operatorname{Sim} 3 \Delta$ aggregates showed enrichment in functions related to metabolic processes and their regulation (Fig. 5C), which is consistent with the aberrant growth phenotypes observed in these mutants.
We hypothesized that genes encoding aggregated proteins may be enriched in codons decoded by hypomodified tRNAs in $\operatorname{Trm} 9 \Delta$, Elp $1 \Delta$ and $\operatorname{Slm} 3 \Delta$ (Table 1 and S10). To validate this hypothesis we used the Anaconda software platform to analyse the genes identified in our aggregated proteomic 
A
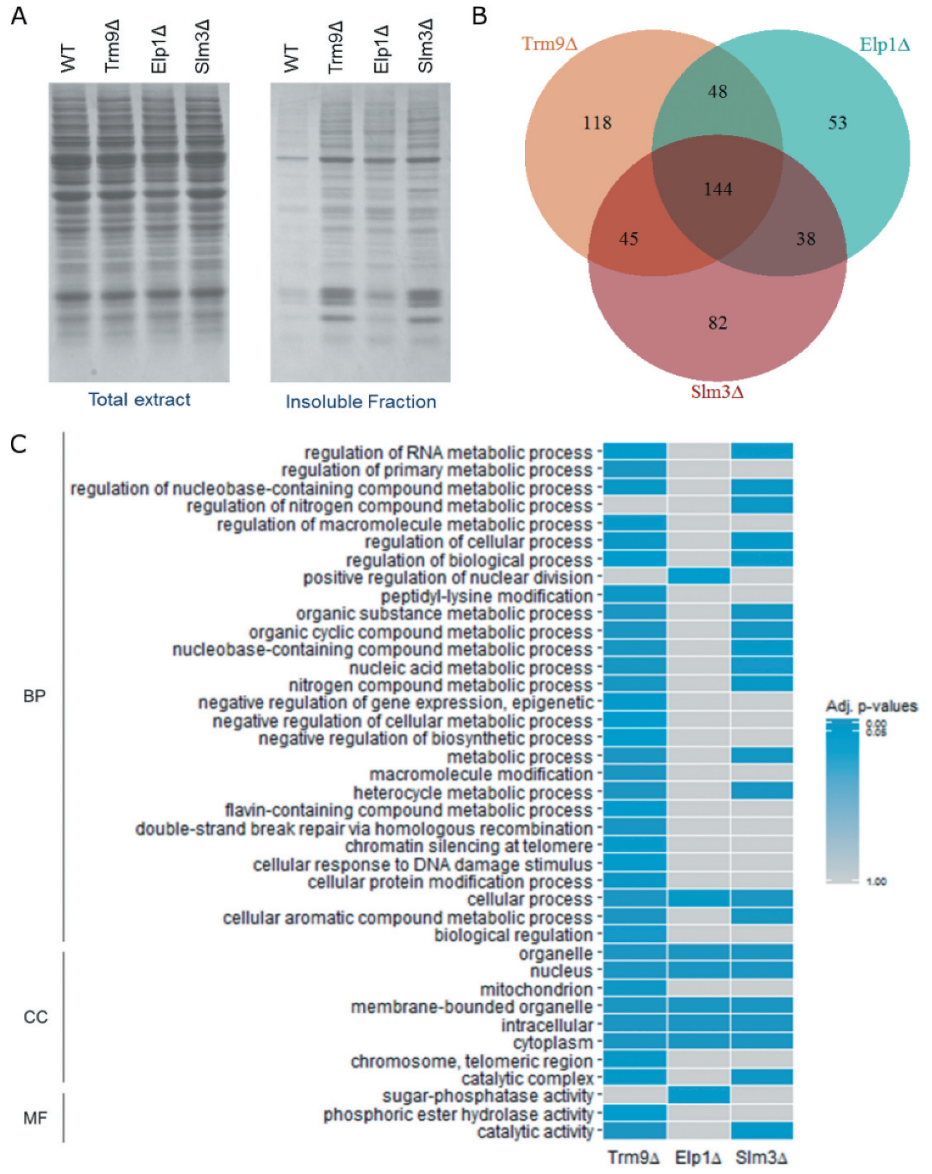

B

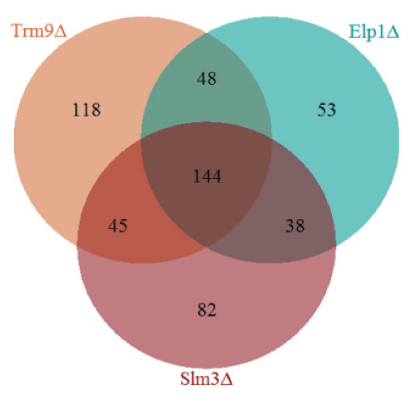

D

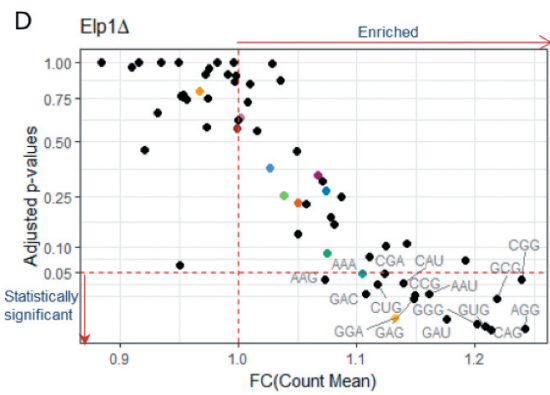

Codons

- aAa

- $A C A$

- aga

- caA

- cCa

- gaA

- gCA

- GGA

- gua

- uca

- uva
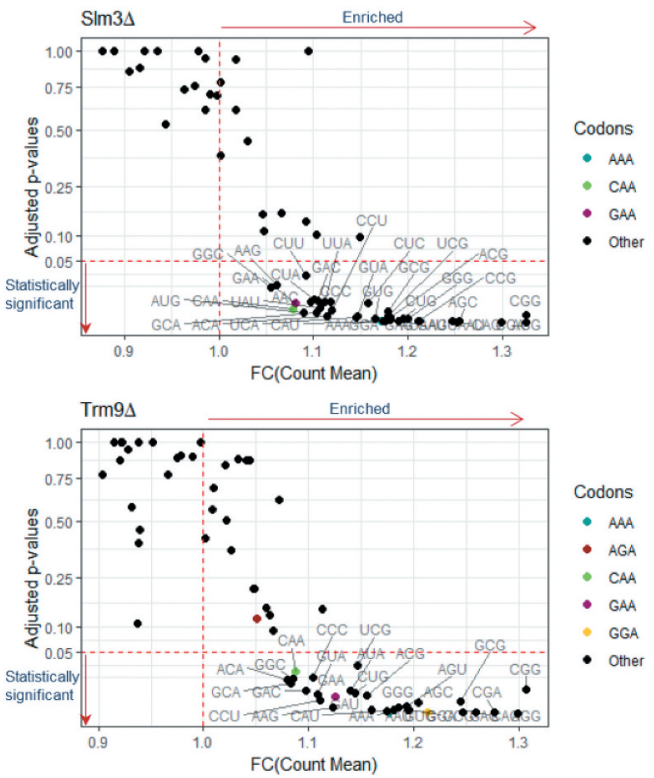

Figure 5. Mutant aggregomes exhibit significant functional enrichments and codon bias. A. SDS-PAGE (14\%) and Coomassie staining was used to visualize total extracts and detergent-insoluble protein aggregates isolated using differential centrifugation of WT and mutants. B. Venn diagram of the overlap between upregulated aggregated proteins identified in selected mutants. C. Heatmap illustrating functional enrichment amongst upregulated proteins identified in selected mutants. Significant enrichments of biological processes amongst detected insoluble proteins were identified using GO data from https://go.princeton.edu/cgi-bin /GOTermFinder. p-values were adjusted with Bonferroni correction and FDR < 5\%. BP: biological process; CC: cellular component; MF: molecular function. D. Aggregated proteins demonstrated significant enrichments in codons decoded by hypomodified tRNAs in mutant cells. Scatter plots show significance of codon usage fold-enrichments in Elp1 $\Delta, \operatorname{SIm} 3 \Delta$ and Trm9 up-regulated proteins relative to reference genome. Significance was determined using the FSA package in R. Dunn (1964) Kruskal-Wallis test was applied for multiple comparisons and $p$-values were adjusted with the Benjamin-Hochberg method. Codons decoded by hypomodified tRNAs are coloured. The red horizontal line indicates a $p$-value of 0.05 and the red vertical indicates a fold change of 1 ; codons below the horizontal line have a statistically significant enrichment and codons on the right of the vertical line are enriched relative to the reference genome. As in Trm9 $9 \Delta$ and SIm3 3 strains, codons decoded by hypomodified tRNAs in Elp1 $\Delta$ demonstrate general overrepresentation amongst aggregates, but these enrichments do not meet our criteria for statistical significance.

Table 1. Codons whose decoding is affected by $\mathbf{U}_{34}$ hypomodification in two-split codon boxes. Loss of $\operatorname{s} / m 3$ perturbs translation of the 3 codons highlighted in green; absence of trm9 affects the decoding of the 7 codons highlighted in blue and green; and deletion of elp1 disrupts decoding of the 8 codons highlighted in red, blue and green.

\begin{tabular}{|c|c|c|c|c|c|}
\hline Codon & Anticodon & Amino acid & Codon & Anticodon & Amino acid \\
\hline CAU & & $\mathrm{His}$ & UUU & & Phe \\
\hline CAC & GUG & & UUC & GmAA & \\
\hline CAA & $\mathrm{mcm}^{5} \mathrm{~s}^{2} \mathrm{UUG}$ & Gln & UUA & $\mathrm{ncm}^{5} \mathrm{UmAA}$ & Leu \\
\hline AAC & GUU & & AGC & GCU & \\
\hline AAA & $\mathrm{mcm}^{5} \mathrm{~s}^{2} \mathrm{UUU}$ & Lys & AGA & $\mathrm{mcm}^{5} \mathrm{UCU}$ & Arg \\
\hline AAG & CUU & & AGG & $\mathrm{CCU}$ & \\
\hline GAG & CUC & & GGG & $\mathrm{CCC}$ & \\
\hline
\end{tabular}

results for biased codon usage [49]. Using functions of the Fish Stock Assessment (FSA) R package, we compared codon frequencies between mutant strains and the reference yeast genome (S288c). This analysis identified significant enrichments of AAA, CAA and GAA codons, whose decoding is affected by $\mathrm{mcm}^{5} \mathrm{~s}^{2} \mathrm{U}_{34}$, in $\operatorname{Trm} 9 \Delta$ and $\operatorname{Slm} 3 \Delta$ mutants; however, these enrichments did not extend to CAA and GAA codons in the Elp1 $\Delta$ strain (Fig. 5D). Previous studies have demonstrated that $\mathrm{mcm}^{5} \mathrm{~s}^{2} \mathrm{U}_{34}$ and $\mathrm{ncm}^{5} \mathrm{U}_{34}$ modifications facilitate preferential decoding of both $\mathrm{A}$ - and, to a lesser 
A
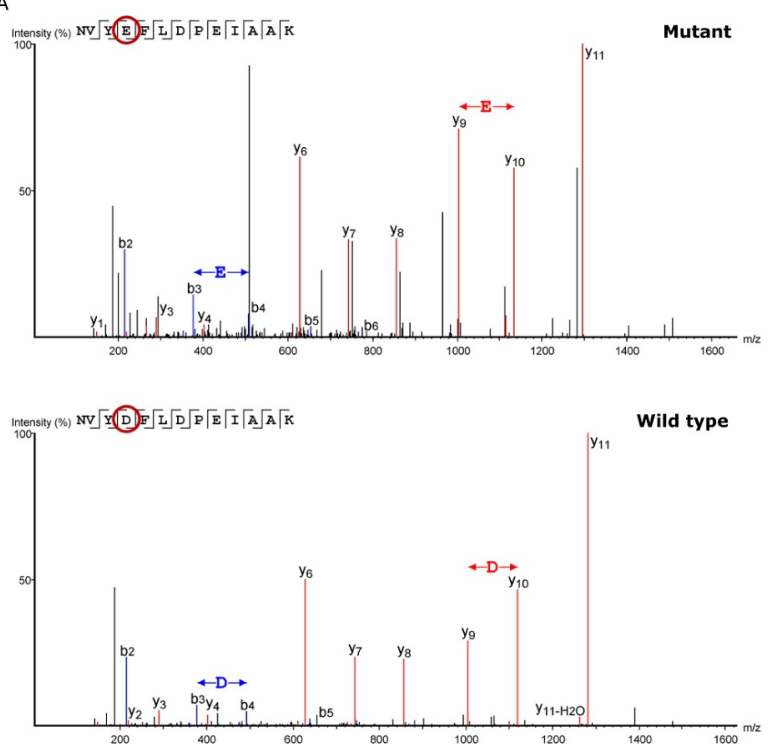

B

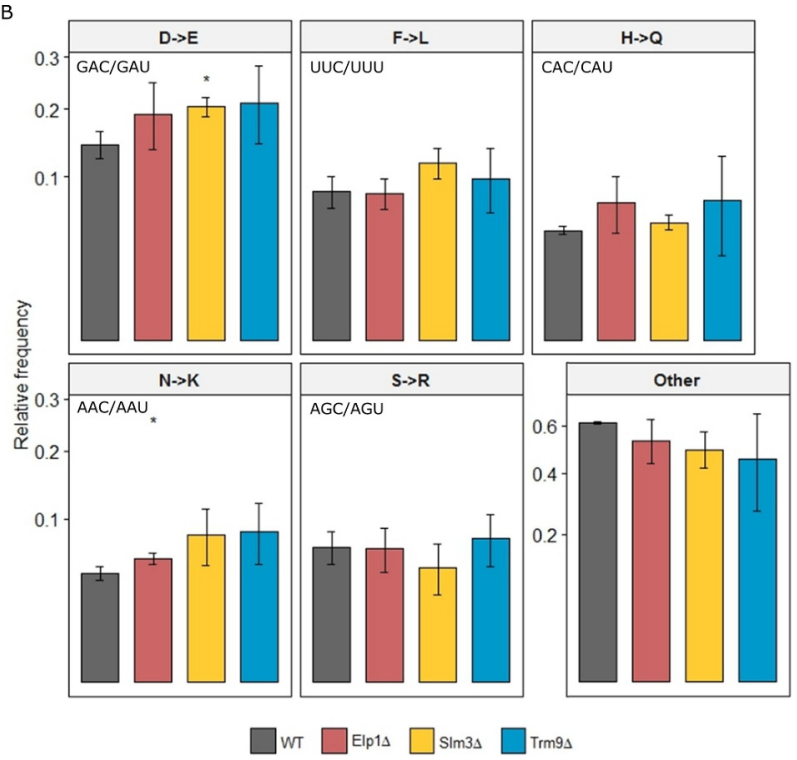

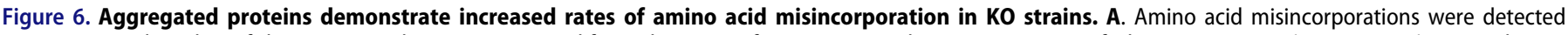

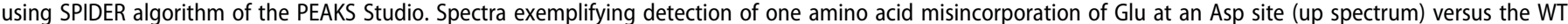
peptide (bottom spectrum). B. Relative frequency of predicted and other amino acid misincorporations in mutant and WT strains.

extent, G-ending codons [50], while $\mathrm{mcm}^{5} \mathrm{U}_{34}$ promotes reading of both A- and G-ending codons, so we decided to analyse genes encoding precipitated proteins for overrepresentation of both A- and G-ending codons. As predicted, codons decoded by $\mathrm{mcm}^{5} \mathrm{U}_{34}$-modified tRNAs (e.g. AGG, GGA and GGG) were statistically enriched, relative to genome average, amongst genes identified from protein aggregates in $\operatorname{Trm} 9 \Delta$ and Elp1 $\Delta$ (Fig. 5D). These results provide evidence that the loss of certain tRNA-modifying enzymes disrupts decoding at related codons and contributes to the precipitation of proteins encoded by codon-biased genes.

\section{Loss of $U_{34}$ modifications increases codon-specific mistranslation}

We next sought to determine whether aggregated proteins were generated by increased translational termination in tRNA-modifying enzyme mutants, or by increased amino acid misincorporation caused by aberrant decoding of certain codons. Since $n \mathrm{~cm}^{5} \mathrm{U}$ and $\mathrm{mcm}^{5} \mathrm{~s}^{2} \mathrm{U}$ have been demonstrated to improve tRNA reading of A-ending codons, while $\mathrm{mcm}^{5} \mathrm{U}$ has been shown to facilitate decoding of both Aand G-ending codons $[10,12,48,50]$, and since wobble base modifications are generally known to prevent tRNA binding to $\mathrm{U}$ - and C-ending codons in two-split codon boxes [51,52], one would expect that mutants harbouring hypomodified tRNA wobble bases experience higher rates of mistranslation. Because $\mathrm{mcm}^{5} \mathrm{~s}^{2} \mathrm{U}_{34}, \mathrm{mcm}^{5} \mathrm{U}_{34}$ and $\mathrm{ncm}^{5} \mathrm{U}_{34}$ are believed to enhance discrimination between cognate and near-cognate codons in mRNA, we hypothesized that mutants lacking these modifications misincorporate amino acids at near cognate sites of $\mathrm{U}$ - and $\mathrm{C}$-ending codons. For example, loss of these modifications could permit decoding of AAA and AAG in place of AAU and AAC codons, leading to amino acid misincorporations at codon sites belonging to arginine $\left(\operatorname{Ser}_{\mathrm{AGC}}\right.$ to $\left.A \mathrm{Ar}_{\mathrm{AGU}}\right)$, glutamic acid (Asp $\mathrm{AAU}_{\mathrm{GA}}$ to $\left.\mathrm{Glu}_{\mathrm{GAC}}\right)$, glutamine ( $\mathrm{His}_{\mathrm{CAU}}$ to $\mathrm{G} \mathrm{ln}_{\mathrm{CAC}}$ ), lysine ( $\mathrm{Asn}_{\mathrm{AAU}}$ to $\mathrm{Lys}_{\mathrm{AAC}}$ ) and leucine (Phe UUU $_{\text {Uu }}$ to Leu $\mathrm{UUC}_{\mathrm{C}}$ ) in the mutant strains (Table 1 and S10).

Computational analysis of our proteomic results identified amino acid misincorporations in insoluble protein fractions of both mutant and WT strains (Fig.s 6A and S4), but found statistically higher rates of Asp-to-Glu and Asn-to-Lys misincorporations in $\operatorname{Slm} 3 \Delta$ and Elp $1 \Delta$ mutants, respectively (Fig. 6B). Furthermore, with few exceptions, we identified predicted amino acid misincorporations at higher rates in aggregated proteins of the mutant strains relative to WT control (Fig. 6B). We also detected amino acid misincorporations in near-cognate codon sites, including increased levels of Asn-to-Lys (AAU/AAC) and Asp-to-Glu (GAC/GAU) conversions in all mutants, as well as other strain- and sitespecific mistranslational events: His-to-Gln (CAU) misincorporations were higher in both $\operatorname{Trm} 9 \Delta$ and $\operatorname{Elp} 1 \Delta$, while Ser-to -Arg (AGC) misincorporations were higher in $\operatorname{Trm} 9 \Delta$ only (Table S11).

We suspected that the absence of $\mathrm{U}_{34}$ modifications might also lead to protein misfolding and degradation by decreasing the decoding speed of a specific subset of codons overrepresented amongst the genes of aggregated proteins. To investigate this possibility, we analysed the distribution of identified amino acid misincorporations across codons (Figure S5), and found that amino acid misincorporation occurred at higher levels at codon positions decoded by hypomodified tRNAs particularly GCA and UUA in Elp1 $\Delta$, and AGA in Trm9 $\Delta$ (Figure S5). Altogether, these findings underscore the critical importance of tRNA modifications in mRNA translational fidelity.

\section{Discussion}

While previous studies of single-gene deletions of tRNAmodifying enzymes have documented primarily subtle 
phenotypes or no measurable phenotype, other reports have shown that disrupting more than one tRNA-modifying gene results in significantly altered cellular physiology, suggesting that epistasis may play a role in regulating tRNA modification profiles in yeast $[32,53,54]$.

In this study, we engineered a genetic screen to assess the functional roles of 70 tRNA-modifying enzymes and identified $5 \mathrm{KO}$ strains with high levels of protein aggregation. The sensitivity of our approach stems from the specificity of experimental techniques optimized for high-throughput fluorescence aggregometry, tRNA isolation, purification, modification profiling and isoacceptor quantification; and panproteomic detection of amino acid misincorporations.

Of the 42 cytosolic yeast tRNAs, 11 have modified uridine at position $34-$ including $n \mathrm{~nm}^{5} \mathrm{U}, \mathrm{ncm}{ }^{5} \mathrm{Um}, \mathrm{mcm}^{5} \mathrm{U}$ and $\mathrm{mcm}^{5} \mathrm{~s}^{2} \mathrm{U}$ [12]. At least 13 proteins are required for the synthesis of $\mathrm{ncm}^{5}$ and $\mathrm{mcm}^{5}$ chains at $U_{34}$ [55], but our genetic screen showed that only Elp1, Kti12, Trm9, Slm3 and Elp3 play significant roles in proteostasis in the growth conditions tested (protein aggregation levels relative to WT: Elp1 $\Delta$ : 4.9-fold; Kti12 2 : 5.1-fold; Trm9 $\Delta$ : 3.4-fold change;: $\operatorname{Slm} 3 \Delta$ : 3.1-fold;: Elp3 $\Delta$ : 6.5 -fold). The $\mathrm{U}_{34}$ modifications levels decreased similarly in Elp $1 \Delta$, Kti $12 \Delta$ and $\operatorname{Trm} 9 \Delta$ strains (Fig. 5B and Supplementary Fig. 4), but the levels of protein aggregation were rather different between them. A possible explanation for this observation could be related to the strong decrease in the abundance of the tRNA ${ }^{\text {Ala }}$ and $\mathrm{tRNA}^{\mathrm{Thr}}$ in Elp1 and Kti12 KO strains (Fig. 5C). - Interestingly, we found that the absence of a given tRNA modification (or its chemical derivatives) affected the abundance of at most two tRNA species, even though many tRNA modifications naturally occur in more than two tRNA isoacceptors. Similar observations have been reported in a survey of 20 sequenced yeast tRNAs lacking $\mathrm{m}^{1} \mathrm{~A}_{58}$, a modification associated with decreased levels of pre-tRNA ${ }_{i}^{\text {Met }}$ [57], and in the selective degradation of tRNA ${ }_{\text {AAC }}^{\mathrm{Val}}$ caused by the absence of $\mathrm{m}^{7} \mathrm{G}_{46}$ and $\mathrm{m}^{5} \mathrm{C}_{49}$, which affects 3 different tRNAs [53].

Most amino acids are encoded by more than one codon (codon redundancy); however, these codons are not used at the same frequency in all genes and influence gene expression in ways that remain poorly understood. Growing evidence suggests that dynamic tRNA modification influences differential use of synonymous codons and may, therefore, regulate the selective translation of specific gene families [54]. For example, independent studies have shown that modifications of the wobble position affect anticodon positioning in the ribosome, allowing for codon-dependent translation of specific transcripts, a mechanism that is particularly relevant for the selective translation of DNA damage response genes $[14,51,58,59]$. Our data showing that the aggresome of the Trm9 $9 \Delta$ strain is enriched in proteins involved in the cellular response to DNA damage, support the involvement of $\operatorname{Trm} 9$ in the stress response to protein- and nuclei acid-damaging agents [58,60]. Similarly, the increase of aggregated proteins associated with metabolic processes and in their regulation in the Slm3 KO strain is consistent with the role of this tRNA-modifying enzyme in mitochondrial tRNA modification and mitochondrial metabolism
$[37,31,61]$. The strong aggregation phenotype observed in this strain highlights the functional connection between the mitochondrial and cytoplasmic proteomes and raises the possibility that $\operatorname{Slm} 3$ may play a role in general proteostasis by affecting, perhaps indirectly, the cytoplasmic proteome. This should be investigated in future studies.

Recent works show that tRNA activity is highly coordinated with mRNA codon demand [62], that some tRNA-modifying enzymes regulate protein expression by modulating translational speed at specific codons $[63,64]$ and that disrupting mRNA decoding dynamics may lead to protein aggregation $[65,66]$. Ribosome footprinting data show that non-modified $\mathrm{U}_{34}$ tRNAs bind poorly to the ribosome, reducing translational speed in a codon-specific manner. In particular, loss of $\mathrm{mcm}^{5}$ modification in Elongator-related mutants was shown to increase ribosome density at AAA, CAA and GAA codons located at the ribosomal A-site $[67,68]$, which agrees with our finding that aggregated proteins in Elp1 $\Delta$ are enriched in AAAbiased genes (Fig. 6D). This study also reported CAA and AAA codon enrichments at ribosomal A-sites ( $\mathrm{Ncs} 6 \Delta$ and Uba $4 \Delta$ mutants) following loss of $s^{2}$ [67], which again agrees with our findings that aggregated proteins in $\operatorname{Sim} 3 \Delta$ are enriched in AAA-, CAA-, GAA- and GAG-biased genes (Fig. 6D).

In vitro studies have shown that wobble base tRNA modifications play a role in translational fidelity [69] and molecular modelling, nuclear magnetic resonance and X-ray data demonstrated that such modifications can alter the geometry of the ribosome-decoding centre, promoting the binding of anticodons to their cognate codons [51,52]. $\mathrm{mcm}^{5} \mathrm{U}_{34}$ and $\mathrm{mcm}^{5} \mathrm{~s}^{2} \mathrm{U}_{34}$ modifications differentiate between cognate and near cognate codons in split codon boxes and optimize codon-anticodon interactions [42,70]. In line with these observations, $U_{34}$ hypomodification of glutamine, glutamic acid and lysine tRNAs reduce the speed of translation in a codon-dependent context [58,71], a phenotype that was reversed by overexpression of the hypomodified tRNAs $[46,54]$. Two hypotheses sprang from these observations: (1) $\mathrm{mcm}^{5} \mathrm{~s}^{2} \mathrm{U}_{34}, \mathrm{mcm}^{5} \mathrm{U}_{34}$ and $\mathrm{ncm}^{5} \mathrm{U}_{34}$ modifications enhance ribosomal binding of anticodons to cognate codons, increasing translational speed in a codon-dependent manner; and (2) the primary role of $\mathrm{mcm}^{5} \mathrm{~s}^{2} \mathrm{U}$ and $\mathrm{mcm}^{5} \mathrm{U}$ modifications is not to reduce misreading of non-cognate codons ending with $\mathrm{U}$ or $\mathrm{C}$ in the split codon boxes, but rather to improve the reading efficiency of cognate codons ending with A. Since $U_{34}$ hypomodification leads to codon-specific translational pausing [66,67], we propose that pausing affects co-translational protein folding (increases misfolding) and increases the rate of amino acid misincorporations, as reported in this study.

The hypomodified tRNAs of the Elp1 $\Delta$ and Elp3 $\Delta$ strains should read 4 codons of both codon and split codon boxes. Previous ribosome occupancy studies showed increased occupancy of only CAC and CAU codons in Elongator mutants [66], while our analysis of protein aggregates in Elp1 $\triangle$ revealed statistically significant enrichments of AAC, AAU, CAU, GAC and GAU (near-cognate codons that can base pair with the Elp1 $\Delta$-hypomodified tRNAs; Fig. 6D and Table S11), suggesting that amino acid misincorporation at near-cognate 
codon sites may contribute to protein misfolding and aggregation.

In conclusion, of the 70 yeast tRNA-modifying enzymes analysed in this work a small subset of those that modify U34 play the most relevant role in proteome homoeostasis by affecting protein folding and synthesis fidelity. Future ribosome profiling studies along with the quantitative tRNA profiling and modification mapping should allow us to investigate mechanistically how codon-specific tRNA modifications, affect proteome stability. It will also be interesting to determine whether protein aggregation profiles change with changing growth conditions.

\section{Acknowledgments}

We thank Eduard Sabidó and Guadalupe Espadas-Garcia from UPF/ CRG Proteomics Unit, and Amanda Del Rosario and Richard P. Schiavoni from MIT Koch Proteomics Core for assistance with proteomics; Rui Fernandes and Francisco Figueiredo from HEMS, IBMC, for technical assistance with transmission electron microscopy; Vera Afreixo and Vera Enes from iBiMED for the statistics support.

\section{Disclosure statement}

No potential conflict of interest was reported by the authors.

\section{Funding}

This work was financially supported by the projects (PTDC/BIA-MIC /31849/2017, PTDC/BIA-MIB/31238/2017; iBiMED through project UID/BIM/04501/2020; funded by FEDER, through (POCI) and by national funds (OE), through FCT/MCTES, and the National Research Foundation of Singapore through the Singapore-MIT Alliance for Research and Technology and the US National Science Foundation [grant number MCB-1412379 to PCD]. JT was supported by a FCT $\mathrm{PhD}$ fellowship [grant number SFRH/BD/86866/2012]. NKD was supported by an NSF Graduate Research Fellowship and an NIH Biotechnology Training Grant at MIT

\section{ORCID}

Joana F Tavares (D) http://orcid.org/0000-0001-7083-6266

Ana Poim (D) http://orcid.org/0000-0003-2947-0308

Inês Sousa (D) http://orcid.org/0000-0001-7062-5346

Ana R. Soares (D) http://orcid.org/0000-0003-4000-7461

Peter C Dedon (D) http://orcid.org/0000-0003-0011-3067

\section{References}

[1] Crick FHC. The origin of the genetic code. J Mol Biol. 1968;38:367-379.

[2] Percudani R, Pavesi A, Transfer OS. RNA gene redundancy and translational selection in saccharomyces cerevisiae. J Mol Biol. 1997;268:322-330.

[3] Hani J, Feldmann H. tRNA genes and retroelements in the yeast genome. Nucleic Acids Res. 1998;26:689-696.

[4] Marck C, Grosjean H. tRNomics: analysis of tRNA genes from 50 genomes of Eukarya, archaea, and bacteria reveals anticodon-sparing strategies and domain-specific features. RNA. 2002;8:1189-1232.

[5] Crick FHC. Codon-anticodon pairing: the wobble hypothesis. J Mol Biol. 1966;19:548-555.

[6] Phizicky EM, Hopper AK. tRNA biology charges to the front. Genes Dev. 2010;24:1832-1860.
[7] Crick FH. The genetic code-yesterday, today, and tomorrow. Cold Spring Harb Symp Quant Biol. 1966;31:1-9.

[8] Gustilo EM, Vendeix FA, Agris PF. tRNA's modifications bring order to gene expression. Curr Opin Microbiol. 2008;11:134-140.

[9] Yokoyama S, Watanabe T, Muraot K, et al. Molecular mechanism of codon recognition by tRNA species with modified uridine in the first position of the anticodon. Biochemistry. 1985;82:4905-4909.

[10] Lim VI, Curran JF. Analysis of codon: anticodon interactions within the ribosome provides new insights into codon reading and the genetic code structure. RNA. 2001;7:942-957.

[11] Sprinzl M, Vassilenko KS. Compilation of tRNA sequences and sequences of tRNA genes. Nucleic Acids Res. 2005;33:D139-40.

[12] Johansson MJO, Esberg A, Huang B, et al. Eukaryotic wobble uridine modifications promote a functionally redundant decoding system. Mol Cell Biol. 2008;28:3301-3312.

[13] Harrington KM, Nazarenko IA, Dix DB, et al. In vitro analysis of translational rate and accuracy with an unmodified tRNA. Biochemistry. 1993;32:7617-7622.

[14] Agris PF. Bringing order to translation: the contributions of transfer RNA anticodon-domain modifications. EMBO Rep. 2008;9:629-635.

[15] Grosjean H. Modification and editing of Rna. Washington, D. C.: Asm Press; 1998.

[16] Motorin Y, Transfer GH. RNA modification. Encycl Life Sci. 2005;1-10.

[17] Grosjean H. Fine-tuning of RNA functions by modifications and editing. Berlin Heidelberg:.Springer-Verlag; 2005.

[18] Jühling F, Mörl M, Hartmann RK, et al. tRNAdb 2009: compilation of tRNA sequences and tRNA genes. Nucleic Acids Res. 2009;37:159-162.

[19] Kramer EB, Hopper AK. Retrograde transfer RNA nuclear import provides a new level of tRNA quality control in Saccharomyces cerevisiae. PNAS. 2013;110:21042-21047.

[20] Perrochia L, Crozat E, Hecker A, et al. In vitro biosynthesis of a universal t6A tRNA modification in archaea and eukarya. Nucleic Acids Res. 2013;41:1953-1964.

[21] Helm M. Post-transcriptional nucleotide modification and alternative folding of RNA. Nucleic Acids Res. 2006;34:721-733.

[22] Chan CTY, Dyavaiah M, DeMott MS, et al. A quantitative systems approach reveals dynamic control of tRNA modifications during cellular stress. PLoS Genet. 2010;6:1-9.

[23] Chan CTY, Ling Y, Pang J, Deng W, Babu IR, Begley TJ, Dedon PC. Reprogramming of tRNA modifications controls the oxidative stress response by codon-biased translation of proteins. Nat Commun. 2012;3.

[24] Jaroensuk J, Atichartpongkul S, Chionh YH, et al. Methylation at position 32 of tRNA catalyzed by $\operatorname{Trm}$ J alters oxidative stress response in pseudomonas aeruginosa. Nucleic Acids Res. 2016;44:10834-10848.

[25] Czerwoniec A, Dunin-Horkawicz S, Purta E, et al. MODOMICS: a database of RNA modification pathways. 2008 update. Nucleic Acids Res. 2009;37:D118-21.

[26] Machnicka MA, Milanowska K, Osman Oglou O, et al. MODOMICS: a database of RNA modification pathways-2013 update. Nucleic Acids Res. 2013;41:D262-7.

[27] Torres AG, Batlle E, Ribas de Pouplana L. Role of tRNA modifications in human diseases. Trends Mol Med. 2014;934:1-9.

[28] Guy MP, Podyma BM, Preston MA, et al. Yeast Trm7 interacts with distinct proteins for critical modifications of the tRNAPhe anticodon loop. RNA. 2012;18:1921-1933.

[29] Motorin Y, Lyko F, Helm M. 5-methylcytosine in RNA: detection, enzymatic formation and biological functions. Nucleic Acids Res. 2010;38:1415-1430.

[30] Kirino Y, Goto Y-I, Campos Y, et al. Specific correlation between the wobble modification deficiency in mutant tRNAs and the clinical features of a human mitochondrial disease. PNAS. 2005;102:7127-7132.

[31] Umeda N, Suzuki T, Yukawa M, et al. RNA-modifying enzymes responsible for the biosynthesis of the wobble base in 
mitochondrial tRNAs: implications for the molecular pathogenesis of human mitochondrial diseases. J Biol Chem. 2005;280:1613-1624.

[32] Chen C, Tuck S, Byström AS. Defects in tRNA modification associated with neurological and developmental dysfunctions in Caenorhabditis elegans elongator mutants. PLoS Genet. 2009;5.

[33] Giaever G, Chu AM, Ni L, et al. Functional profiling of the saccharomyces cerevisiae genome. Nature. 2002;418:387-391.

[34] Gietz RD, Woods RA. Yeast transformation by the LiAc/SS carrier DNA/PEG method. In: Xiao W, editor. Yeast Protocols. New Jersey:: Humana Press; 2006. p. 107-120.

[35] Santos MAS, Perreau VM, Tuite MF. Transfer RNA structural change is a key element in the reassignment of the CUG codon in Candida albicans. Embo J. 1996;15:5060-5068.

[36] Su D, Chan CTY, Gu C, et al. Quantitative analysis of ribonucleoside modifications in tRNA by HPLC-coupled mass spectrometry. Nat Protoc. 2014;9:828-841.

[37] Honda S, Shigematsu M, Morichika K, et al. Four-leaf clover qRT-PCR: A convenient method for selective quantification of mature tRNA. RNA Biol. 2015;12:501-508.

[38] Pfaffl MW. Horgan G W, Dempfle L. Relative expression software tool (REST@) for group-wise comparison and statistical analysis of relative expression results in real-time PCR. Nucleic Acids Res. 2002;30.

[39] Koplin A, Preissler S, Llina Y, et al. A dual function for chaperones SSB-RAC and the NAC nascent polypeptide-associated complex on ribosomes. J Cell Biol. 2010;189:57-68.

[40] Fujita K, Kawai R, Iwahashi H, et al. Hsp104 responds to heat and oxidative stress with different intracellular localization in saccharomyces cerevisiae. Biochem Biophys Res Commun. 1998;248:542-547.

[41] Erjavec N, Larsson L, Grantham J, et al. Accelerated aging and failure to segregate damaged proteins in Sir2 mutants can be suppressed by overproducing the protein aggregation-remodeling factor Hsp104p. Genes Dev. 2007;21:2410-2421.

[42] Kalhor HR, Clarke S. Novel methyltransferase for modified uridine residues at the wobble position of tRNA. Mol Cell Biol. 2003;23:9283-9292.

[43] Huang BO, Johansson MJO, Byström AS. An early step in wobble uridine tRNA modification requires the Elongator complex. 2005;424-436.

[44] Chen C, Huang B, Anderson JT, et al. Unexpected accumulation of $\mathrm{ncm} 5 \mathrm{U}$ and $\mathrm{ncm} 5 \mathrm{~S} 2 \mathrm{U}$ in a trm 9 mutant suggests an additional step in the synthesis of mcm5U and mcm5S2U. PLoS One. 2011;6.

[45] Petrakis TG, TMM S, Erdjument-Bromage H, et al. Physical and functional interaction between elongator and the chromatin-associated Kti12 protein. J Biol Chem. 2005;280: 19454-19460.

[46] Esberg A, Huang B, Johansson MJO, et al. Elevated levels of two tRNA species bypass the requirement for elongator complex in transcription and exocytosis. Mol Cell. 2006;24:139-148.

[47] Klassen R, Grunewald P, Thüring KL, et al. Loss of anticodon wobble uridine modifications affects tRNALys function and protein levels in Saccharomyces cerevisiae. PLoS One. 2015;10:1-17.

[48] Björk GR, Huang B, Persson OP, et al. A conserved modified wobble nucleoside $(\mathrm{mcm} 5 \mathrm{~s} 2 \mathrm{U})$ in lysyl-tRNA is required for viability in yeast. Rna. 2007;13:1245-1255.

[49] Moura G, Pinheiro M, Silva R, et al. Comparative context analysis of codon pairs on an ORFeome scale. Genome Biol. 2005;6.

[50] Grosjean H, Westhof E. An integrated, structure- and energy-based view of the genetic code. Nucleic Acids Res. 2016;44:8020-8040.

[51] Agris PF. Decoding the genome: a modified view. Nucleic Acids Res. 2004;32:223-238.
[52] Durant PC, Bajji AC, Sundaram M, et al. Structural effects of hypermodified nucleosides in the escherichia coli and human tRNA. Biochemistry. 2005;44:8078-8089.

[53] Alexandrov A, Chernyakov I, Gu W, et al. Rapid tRNA decay can result from lack of nonessential modifications. Mol Cell. 2006;21:87-96.

[54] Bauer F, Matsuyama A, Candiracci J, et al. Translational control of cell division by Elongator. Cell Rep. 2012;1:424-433.

[55] Ranjan N, Rodnina MV. tRNA wobble modifications and protein homeostasis. Translation. 2016;4:e1143076.

[56] Kadaba S, Wang X, Anderson JT. Nuclear RNA surveillance in Saccharomyces cerevisiae: trf4p-dependent polyadenylation of nascent hypomethylated tRNA and an aberrant form of $5 \mathrm{~S}$ rRNA. RNA. 2006;12:508-521.

[57] Anderson J, Phan L, Cuesta R, et al. The essential Gcd10pGcd14p nuclear complex is required for 1-methyladenosine modification and maturation of initiator methionyl-tRNA. Genes Dev. 1998;12:3650-3662.

[58] Begley U, Dyavaiah M, Patil A, et al. Trm9-catalyzed tRNA modifications link translation to the DNA damage response. Mol Cell. 2007;28:860-870.

[59] Huang B, Lu J, Byström AS. A genome-wide screen identifies genes required for formation of the wobble nucleoside 5-methoxycarbonylmethyl-2-thiouridine in Saccharomyces cerevisiae. RNA. 2008;14:2183-2194.

[60] Chen C, Huang B, Eliasson M, et al. Elongator complex influences telomeric gene silencing and DNA damage response by its role in wobble uridine tRNA modification. PLoS Genet. 2011;7.

[61] Wu Y, Wei FY, Kawarada L, et al. Mtul-mediated thiouridine formation of mitochondrial tRNAs is required for mitochondrial translation and is involved in reversible infantile liver injury. PLoS Genet. 2016;12:1-19.

[62] Maraia RJ, Iben JR. Different types of secondary information in the genetic code. RNA. 2014;20:977-984.

[63] Novoa EM, Ribas de Pouplana L. Speeding with control: codon usage, tRNAs, and ribosomes. Trends Genet. 2012;28:574-581.

[64] Ff V C, Le Guyon S, Hughes KT. The effects of codon context on in vivo translation speed. PLoS Genet. 2014;10:e1004392.

[65] Klassen R, Ciftci A, Funk J, et al. tRNA anticodon loop modifications ensure protein homeostasis and cell morphogenesis in yeast. Nucleic Acids Res. 2016;44:10946-10959.

[66] Nedialkova DD, Leidel SA. Optimization of codon translation rates via tRNA modifications maintains proteome integrity. Cell. 2015;161:1606-1618. [Internet]. Available from. .

[67] Zinshteyn B, Gilbert WV. Loss of a conserved tRNA anticodon modification perturbs cellular signaling. PLoS Genet. 2013;9.

[68] Chou HJ, Donnard E, Gustafsson HT, et al. Transcriptome-wide analysis of roles for tRNA modifications in translational regulation. Mol Cell [Internet]. 2017;68:978-992.e4.

[69] Yarian C, Townsend H, Czestkowski W, et al. Accurate translation of the genetic code depends on tRNA modified nucleosides. J Biol Chem. 2002;277:16391-16395.

[70] Patil A, Chan CTY, Dyavaiah M, et al. Translational infidelity-induced protein stress results from a deficiency in Trm9-catalyzed tRNA modifications. RNA Biol. 2012;9:990-1001.

[71] Ashraf SS, Sochacka E, Cain R, et al. Single atom modification (O -> S) of tRNA confers ribosome binding. RNA. 1999;5:188-194.

[72] Huh KW, Falvo VJ, Gerke CL, et al. Global analysis of protein localization in budding yeast. Nat. 2003;425:686-691. Available from: http://www.nature.com.ezproxyhost.library.tmc.edu/nature/ journal/v425/n6959/abs/nature02026.html\%0Ahttp://www.ncbi. nlm.nih.gov/pubmed/14562095 